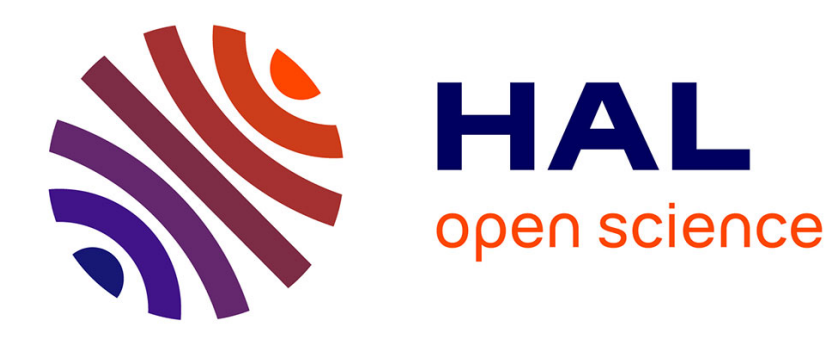

\title{
Maxwell Garnett approximation (advanced topics): tutorial
}

\author{
Vadim A Markel
}

\section{To cite this version:}

Vadim A Markel. Maxwell Garnett approximation (advanced topics): tutorial. Journal of the Optical Society of America. A Optics, Image Science, and Vision, 2016, 33 (11), pp.2237 - 2255. 10.1364/JOSAA.33.002237 . hal-01389181

\section{HAL Id: hal-01389181 https://hal.science/hal-01389181}

Submitted on 16 Nov 2016

HAL is a multi-disciplinary open access archive for the deposit and dissemination of scientific research documents, whether they are published or not. The documents may come from teaching and research institutions in France or abroad, or from public or private research centers.
L'archive ouverte pluridisciplinaire $\mathbf{H A L}$, est destinée au dépôt et à la diffusion de documents scientifiques de niveau recherche, publiés ou non, émanant des établissements d'enseignement et de recherche français ou étrangers, des laboratoires publics ou privés. 


\title{
Maxwell Garnett approximation (advanced topics): tutorial
}

\author{
VADIM A. MARKEL * \\ Aix-Marseille Université, CNRS, Centrale Marseille, Institut Fresnel UMR 7249, 13013 Marseille, France \\ Corresponding author: vmarkel@fresnel.fr \\ Compiled November 16, 2016
}

In the second part of this tutorial we consider several advanced topics related to the Maxwell Garnett approximation.

OCIS codes: (000.1600) Classical and quantum physics; (160.0160) Materials.

http://dx.doi.org/10.1364/JOSAA.33.002237

\section{INTRODUCTION}

This is the second part of the tutorial on Maxwell Garnett approximation. The first, introductory part was published in [1] and we will refer to the equations of [1] in the form (FP\#), where \# is the equation number.

In the second part, several more advanced topics will be discussed. The overall goal is to justify and generalize the Maxwell Garnett approximation and to place it more solidly in the context of modern classical electrodynamics of continuous media. In particular, we will include into consideration the effects of finite frequencies and electromagnetic interaction of inclusions. We will also develop a rigorous homogenization theory for periodic composites. We will see that the Maxwell Garnett mixing formula is obtained from this result by neglecting a term that is responsible for the interaction of inclusions.

We will start with laying out in section 2 a general theoretical framework of the macroscopic Maxwell's equations in continuous media. This old topic has attracted renewed attention recently $[2,3]$. Traditionally, the derivation of macroscopic Maxwell's equations from the microscopic ones was done primarily for pedagogical purposes and the averaging procedure typically involved in such derivations was not used constructively. However, the development of modern computational approaches to the microscopic theory and, especially, the emergence of the DFT-based ab initio methods for computing the microscopic electromagnetic quantities have motivated re-examination of some basic assumptions.

One important idea that requires a fresh look is the classical interpretation of the polarization and magnetization fields as the volume densities of the electric and magnetic dipole moments. Ab initio calculations of the microscopic induced charge density in some solids such as crystalline silicon have shown

*On leave from the Department of Radiology, University of Pennsylvania, Philadelphia, Pennsylvania 19104, USA; vmarkel@mail.med.upenn.edu that this interpretation can be incorrect [4]. In section 3, we discuss the range of applicability of the classical interpretation and identify two main reasons for its breakdown: charge leakage through the cell boundaries (in crystalline materials) and accumulation of a non-negligible electromagnetic phase between the neighboring cells. The two causes for the classical interpretation breakdown become important in different physical situations. Charge leakage can be relevant for natural materials in which the elementary cells are microscopic but not for macroscopic composites. On the other hand, the phase shift plays almost no role in natural materials but can come to the fore in the macroscopic homogenization theories.

In section 4 we consider propagation of waves on threedimensional cubic lattices of polarizable particles. It is shown that the Clausius-Mossotti relation follows from the law of dispersion for such waves in the long wavelength approximation. The Maxwell Garnett mixing formula is then obtained from the Clausius-Mossotti relation if we assume that the particles are small dielectric spheres and use the appropriate expression for their polarizabilities. We also discuss briefly what happens beyond the long wavelength approximation and, in particular, give a few leads to the extended homogenization theories that include dynamic corrections and effects of nonlocality.

A drawback of the point-particle model is that it does not allow one to account for the shape and physical size of the inclusions in a mathematically-consistent manner. In section 5, we overcome this limitation by considering a general threedimensional photonic crystal. A rigorous homogenization theory is obtained in the long wavelength limit. The resulting mixing formula is very similar to Maxwell Garnett's but contains an additional term, which is responsible for the electromagnetic interaction of inclusions. This term also allows one to compute tensorial effective permittivities of anisotropic composites.

In section 6, we show several numerical illustrations of the rigorous homogenization formula derived in section 5 . Here 
we consider various two-component composites consisting of the same materials (a conductor and a dielectric) as were used in the first part of this tutorial to illustrate the Wiener and Bergman-Milton bounds. The results are compared to the Maxwell Garnett's and Bruggeman's predictions and it is shown that the Maxwell Garnett approximation is quite robust for isotropic composites, albeit we do not consider strong resonance effects that can render simple mixing formulas grossly inaccurate. However, in the case of anisotropic composites, Maxwell Garnett's predictions are much less robust even in the absence of strong resonances.

Consistently with the first part of this tutorial, we will use the Gaussian system of units.

\section{MACROSCOPIC MAXWELL'S EQUATIONS IN CON- TINUOUS MEDIA}

The starting point for our exposition is the set of microscopic Maxwell's equations for the electric and magnetic fields $\mathbf{E}$ and B and the electric current $\mathbf{J}$,

$$
\nabla \times \mathbf{B}=\frac{1}{c} \frac{\partial \mathbf{E}}{\partial t}+\frac{4 \pi}{c} \mathbf{J}, \quad \nabla \times \mathbf{E}=-\frac{1}{c} \frac{\partial \mathbf{B}}{\partial t},
$$

where $c$ is the speed of light in vacuum. The system (1) is supplemented by the continuity equation

$$
\frac{\partial \rho}{\partial t}+\nabla \cdot \mathbf{J}=0
$$

where $\rho$ is the density of electric charge. The two equations $\nabla \cdot \mathbf{E}=4 \pi \rho$ and $\nabla \cdot \mathbf{B}=0$ are not independent but follow from (1) and (2).

The fields $\mathbf{E}$ and $\mathbf{B}$ are fundamental in the electromagnetic theory because they appear in the expression for the Lorentz force $\mathbf{F}$ acting on a point charged particle, viz,

$$
\mathbf{F}=q \mathbf{E}+\frac{q}{c} \mathbf{v} \times \mathbf{B} .
$$

In classical physics, electric and magnetic fields are measurable only through the action of the Lorentz force [5].

Some part of the electric current $\mathbf{J}$ can be viewed as a known and externally-controlled source. One example is a radiating antenna, which is fed in a prescribed way. We denote this term by $\mathbf{J}_{\text {ext }}$ and refer to it as to the external current. The electromagnetic fields created by Jext will exert the force (3) and set the charges of any material object in motion. This will create an induced current $\mathbf{J}_{\text {ind }}$ in that object. The total current everywhere in space is then given by

$$
\mathbf{J}(\mathbf{r}, t)=\mathbf{J}_{\text {ext }}(\mathbf{r}, t)+\mathbf{J}_{\text {ind }}(\mathbf{r}, t) .
$$

In any sensible application of the theory, the external and the induced currents do not overlap spatially. Consequently, $\mathbf{J}_{\text {ext }}(\mathbf{r}, t)$ does not overlap with the medium. One can consider a more general case mathematically, but such models do not describe the physical reality.

The induced current will create additional electromagnetic fields, which will, in turn, act back on the moving charges. Even if we can neglect the back-action of the induced fields on the external source, this interaction will result in a big unsolvable set of nonlinear equations. To complicate things further, it is known that any set of charged particles can not hold itself together by the forces of the form (3) if we apply the Newtonian laws of motion. Therefore, stability of matter can not be understood from classical first principles.
Naturally, one wishes to develop an electromagnetic theory of continuous media that avoids these complications. This goal is achieved by the macroscopic Maxwell's equation. In a typical textbook exposition, these equations are obtained by averaging the microscopic equations (1) over a physically small volume. The derivation is based on the observation that $\langle\nabla \times \mathbf{E}\rangle=\nabla \times\langle\mathbf{E}\rangle$ and similarly for other fields, where

$$
\langle f(\mathbf{r})\rangle=\frac{1}{D} \int_{\mathbb{D}} f(\mathbf{r}+\mathbf{s}) d^{3} s,
$$

$\mathbb{D}$ is a small region of space around the origin and $D$ is its volume. We then replace all quantities in (1) by their averages. The nontrivial task in this case is to compute $\left\langle\mathbf{J}_{\text {ind }}\right\rangle$, which describes the response of the medium to the electromagnetic fields.

Unfortunately, there exist several problems with the averaging approach. First, for the method to be mathematically sound, we need to make sure that the integrals of the form (5) exist for all quantities of interest and are independent of the shape of D. But the field of a truly point charge is not integrable and therefore it can not be averaged. This problem is not rectified by introducing any number of point charges or by excluding small spherical regions around each charge from integration (this does not suppress the dependence on $\mathbb{D}$ ). The only solution to the divergence problem is to introduce a continuous density of charge. In particular, the quantum-mechanical DFT and the classical hydrodynamic model operate with continuous densities, which are already in some sense averages. But these smooth functions are not obtained by actual averaging of some (generally, unknown) microscopic densities; they are simply postulated to exist and are computed according to some approximations.

Secondly, the microscopic quantities of interest are very difficult if not impossible to compute in continuous media. Of course, there exist some simple models such as the Lorentz or the Drude model. However, these models describe adequately only some special cases and, moreover, they do not employ averaging of the field of point charges. In the first part of this tutorial, we have averaged the field of elementary dipoles (in section 2B). This is a somewhat better-defined procedure albeit not without its own mathematical ambiguities, which we have encountered while considering anisotropy in section 4 .

We can conclude that the operation of microscopic field averaging is a purely formal procedure; it is not constructive in the sense that it does not yield any useful predictions. The averaging is simply postulated for pedagogical reasons and not used for anything. Instead, a phenomenological approach to constructing the macroscopic theory is implicitly used. Indeed, all we need to "derive" the macroscopic Maxwell's equations are the following three postulates:

(i) The medium is a true continuum, possibly, with sharp boundaries or interfaces where the properties can jump abruptly.

(ii) The external source excites a continuous density of induced current $\mathbf{J}_{\text {ind }}$ (possibly, with singular contributions at the surfaces of discontinuity), which is a functional of the fundamental electromagnetic fields.

(iii) The induced current is zero in vacuum.

In the case of local and linear media, we can describe the functional dependence of postulate (ii) by the equation

$$
\mathbf{J}_{\text {ind }}=\frac{\partial \mathbf{P}}{\partial t}+c \nabla \times \mathbf{M}
$$


where

$$
\begin{aligned}
\mathbf{P}(\mathbf{r}, t) & =\int_{0}^{\infty} f_{e}(\mathbf{r}, \tau) \mathbf{E}(\mathbf{r}, t-\tau) d \tau, \\
\mathbf{M}(\mathbf{r}, t) & =\int_{0}^{\infty} f_{m}(\mathbf{r}, \tau) \mathbf{B}(\mathbf{r}, t-\tau) d \tau .
\end{aligned}
$$

Here $f_{e}(\mathbf{r}, \tau)$ and $f_{m}(\mathbf{r}, \tau)$ are the electric and magnetic influence functions (in general, symmetric tensors). We can say that (7) is causal and local: for example, $\mathbf{J}(\mathbf{r}, t)$ depends only on the electric field at the same point $\mathbf{r}$ (locality) and at the moments of time $t^{\prime} \leq t$ (causality). Note that according to the Postulate (iii), $f_{e}(\mathbf{r}, \tau)=f_{m}(\mathbf{r}, \tau)=\mathbf{P}(\mathbf{r}, t)=\mathbf{M}(\mathbf{r}, t)=0$ if $\mathbf{r}$ is in vacuum.

Now we need to make a few important comments.

(i) Expressions (6),(7) are not the most general form of a linear functional relating the induced current to the fundamental fields. In bi-isotropic and bi-anisotropic media, (7) is replaced by a more general relation in which $\mathbf{P}$ is coupled to $\mathbf{B}$ and $\mathbf{M}$ is coupled to E. However, (6),(7) are of the most general form if we restrict consideration to media that are completely characterizable by two symmetric tensors $\epsilon$ and $\mu$ (the dielectric permittivity and magnetic permeability, defined below). Physically, our assumptions mean that the medium is reciprocal and non-chiral. There exists a subtle point: the gyrotropic (magnetooptical) effect is described by a non-symmetric tensor $\epsilon$; it may seem that this phenomenon can be formally described by (6),(7). In fact, the off-diagonal elements of $\epsilon$ in gyrotropic media are proportional to the amplitude of a stationary magnetic field and (6),(7) do not allow for such a dependence. So our description does not include gyrotropy and non-reciprocity caused by a stationary magnetic field.

(ii) Just like the microscopic Maxwell's equations (1), expressions (6),(7) are invariant under coordinate inversion. Recall that the true vectors such as $\mathbf{E}, \mathbf{J}$ and $\mathbf{P}$ change sign while the pseudo-vectors $\mathbf{B}, \mathbf{M}$ are invariant under coordinate inversion. However, invariance under time inversion is lost in the macroscopic theory. Time inversion can be understood as an instantaneous change of direction of the classical velocities of all particles (we do not discuss here antimatter and other quantum concepts). The fields $\mathbf{J}$ and $\mathbf{B}$ and the time derivatives such as $\partial \mathbf{E} / \partial t$ change sign under time inversion while $\mathbf{E}$ and $\rho$ are invariant. Invariance under time inversion is lost in the macroscopic equations because $\mathbf{P}(t)$ in (7a) depends on $\mathbf{E}\left(t^{\prime}\right)$ for all moments $t^{\prime}$ such that $t^{\prime}<t$. If this dependence was local, e.g., $\mathbf{P}(t)=\chi_{e} \mathbf{E}(t)$ (and similarly for $\mathbf{M}$ ), the macroscopic equations would have been time-reversible. It is well known that temporal nonlocality of (7), also known as temporal or frequency dispersion, is a phenomenological way of accounting for thermodynamically irreversible processes such as absorption.

(iii) The familiar equation formalizing the Ohm's law, $\mathbf{J}_{\text {ind }}=$ $\sigma \mathbf{E}$, where $\sigma$ is the conductivity, is a special case of (6),(7). Analysis is most straightforward in the frequency domain, but we can also use the equation $\partial \mathbf{P} / \partial t=\sigma \mathbf{E}$ to write $\mathbf{P}(t)=$ $\sigma \int_{-\infty}^{t} \mathbf{E}\left(t^{\prime}\right) d t^{\prime}$; comparing this to (7a), we find that $f_{e}(\tau)=\sigma$, that is, $f_{e}(\tau)$ is independent of $\tau$ for conductors. We can also write $f_{e}(\tau)=\sigma \Theta(\tau)$ where $\Theta(x)$ is the step function and expand integration in (7a) to the whole real axis. Note that Ohm's law is a prime example of time-reversibility breaking since the fields $\mathbf{J}$ and $\mathbf{E}$ transform differently under time inversion.

(iv) We have used the same letters E, B, etc., to denote the microscopic and macroscopic electromagnetic fields. We thus break off with the tradition to denote the microscopic fields by small letters such as $\mathbf{e}$ or $\mathbf{b}$. The latter approach usually implies that $\mathbf{E}=\langle\mathbf{e}\rangle$, etc. We do not follow the tradition since we associate a given notation with a physical quantity it represents rather than with a particular approximation. Thus, E is the electric field that appears in the expression for the Lorentz force (3), and the macroscopic theory simply offers us a mathematicallytractable approximation for computing this quantity. Moreover, the small-letter notations are not really used for anything: as soon as the averaging operation is formally introduced, all calculations are performed with the large-letter quantities, including the calculations of physical measurables that are quadratic in the fields. These calculations bear no trace of the averaging procedure and, therefore, the existence or non-existence of the more fundamental little-letter fields is of no observable consequence. However, we will use below the notations $\varrho$ and $\mathbf{j}$ for the microscopic densities of charge and current. These quantities are introduced in various theoretical models of continuous media.

(v) The term $c \nabla \times \mathbf{M}$ can be viewed as a spatially-nonlocal correction to the purely local term $\partial \mathbf{P} / \partial t$. We can envisage terms with higher-order derivatives or a more general nonlocal functional dependence. In this case, the linear relation between $\mathbf{J}_{\text {ind }}$ and $\mathbf{E}$ becomes of a very general form $\mathbf{J}_{\text {ind }}(\mathbf{r}, t)=$ $\int d^{3} r^{\prime} \int_{\left|\mathbf{r}-\mathbf{r}^{\prime}\right| / c}^{\infty} d \tau f\left(\mathbf{r}, \mathbf{r}^{\prime}, \tau\right) \mathbf{E}\left(\mathbf{r}^{\prime}, t-\tau\right)$ where $f$ can have singularities that describe derivatives of arbitrary order. This description requires detailed knowledge of the kernel $f\left(\mathbf{r}, \mathbf{r}^{\prime}, \tau\right)$. It is often implied that $f$ is a function of the shift $\mathbf{r}-\mathbf{r}^{\prime}$, but this is true only sufficiently far from the boundaries and interfaces. If $f$ is known only far from the interfaces (as is usually the case), introduction of the so-called additional boundary conditions $\left(A B C^{\prime} s\right)$ and additional medium parameters is required. In natural materials, the effect of nonlocality, although in principle unavoidable, is very weak, and $f$ quickly approaches zero for the shifts $\left|\mathbf{r}-\mathbf{r}^{\prime}\right|$ that are significantly larger than the atomic scale. On the other hand, nonlocality can result in some physical effects (such as optical activity), which are not present in purely local materials. A detailed exposition of the nonlocal optics of crystals is given in [6]. In macroscopic composites, nonlocality can play a much more profound role, see Sec. 4.E below.

(vi) Substitution of (7) into (2) results in the expression for the induced charge density,

$$
\rho_{\text {ind }}(\mathbf{r}, t)=-\nabla \cdot \mathbf{P}(\mathbf{r}, t) .
$$

We could have added to the right-hand side of (8) any timeindependent function $\rho_{0}(\mathbf{r})$. It is usually assumed that the medium is locally and globally neutral in the absence of external excitation so that $\rho_{0}=0$. We have used a similar assumption in Point (iii) above to determine the constant of integration. However, in some cases, this assumption is not valid. Examples include charged objects and segnetoelectrics. In what follows, we will assume that $\rho_{0}=0$.

(vii) The quantities $\mathbf{P}$ and $\mathbf{M}$ define uniquely $\mathbf{J}_{\text {ind }}$ through Eq. (6) but the inverse is not true. Indeed, the pair $\left(\mathbf{P}^{\prime}, \mathbf{M}^{\prime}\right)$, where $\mathbf{P}^{\prime}=\mathbf{P}+\nabla \times \mathbf{F}$ and $\mathbf{M}^{\prime}=\mathbf{M}-\partial \mathbf{F} / \partial t$ yield the same $\mathbf{J}_{\text {ind }}$ as the pair $(\mathbf{P}, \mathbf{M})$. Here $\mathbf{F}$ is an arbitrary pseudo-vector field. This fact has historically led to the proposition that, in order to build the correct macroscopic theory, one must resort to some additional conditions to define $\mathbf{P}$ and $\mathbf{M}$ uniquely [\$6 7]. However, in the phenomenological approach described here, one does not really need to determine $\mathbf{P}$ and $\mathbf{M}$ from a given $\mathbf{J}_{\text {ind }}$. Instead, the physically-relevant problem is to determine the functions $f_{e}(\tau)$ and $f_{m}(\tau)$ for a given material. This can be done uniquely by performing several measurements of the 
transmitted and reflected field in some simple geometries such as a slab. A particular example of an experimental technique for determining the dielectric function of a homogeneous material is called ellipsometry [8]. Once $f_{e}(\tau)$ and $f_{m}(\tau)$ (in practice, their Fourier transforms with respect to $\tau$ ) have been found, any boundary-value problem can be solved uniquely for any illumination and any shape of the sample. Correspondingly, the fields $\mathbf{P}$ and $\mathbf{M}$ are also defined uniquely in each particular case. Therefore, we do not need to apply the additional condition that $\mathbf{P}$ and $\mathbf{M}$ are the densities of electric and magnetic dipole moments. In fact, we will see that this condition does not hold in general.

We now substitute (6) into (4) and the result into the righthand side of (1) and obtain the macroscopic equations

$$
\nabla \times \mathbf{H}=\frac{1}{c} \frac{\partial \mathbf{D}}{\partial t}+\frac{4 \pi}{c} \mathbf{J}_{\mathrm{ext}}, \quad \nabla \times \mathbf{E}=-\frac{1}{c} \frac{\partial \mathbf{B}}{\partial t},
$$

where we have defined two auxiliary fields

$$
\mathbf{D}=\mathbf{E}+4 \pi \mathbf{P}, \quad \mathbf{H}=\mathbf{B}-4 \pi \mathbf{M}
$$

The second equations in (1) and (9) are the same.

To complete the construction of the theory, we transform the macroscopic Maxwell's equations to the frequency domain. Let us assume that the source $\mathbf{J}_{\text {ext }}(\mathbf{r}, t)$ is strictly monochromatic. Then we can write

$$
\mathbf{J}_{\text {ext }}(\mathbf{r}, t)=\operatorname{Re}\left[\mathbf{J}_{\text {ext }}(\mathbf{r}) e^{-i \omega t}\right], \quad \mathbf{E}(\mathbf{r}, t)=\operatorname{Re}\left[\mathbf{E}(\mathbf{r}) e^{-i \omega t}\right]
$$

and similarly for all other fields. We then obtain from (7),(10):

$$
\mathbf{D}(\mathbf{r})=\epsilon(\mathbf{r}) \mathbf{E}(\mathbf{r}), \quad \mathbf{B}(\mathbf{r})=\mu(\mathbf{r}) \mathbf{H}(\mathbf{r}),
$$

where

$$
\begin{aligned}
\epsilon(\mathbf{r}) & =1+4 \pi \int_{0}^{\infty} \chi_{e}(\mathbf{r}, \tau) \exp (i \omega \tau) d \tau, \\
\mu^{-1}(\mathbf{r}) & =1+4 \pi \int_{0}^{\infty} \chi_{m}(\mathbf{r}, \tau) \exp (i \omega \tau) d \tau .
\end{aligned}
$$

Substituting (11),(12) into (9), we arrive at

$$
\begin{aligned}
& \nabla \times \mathbf{H}(\mathbf{r})=-i k \epsilon(\mathbf{r}) \mathbf{E}(\mathbf{r})+\frac{4 \pi}{c} \mathbf{J}_{\text {ext }}(\mathbf{r}), \\
& \nabla \times \mathbf{E}(\mathbf{r})=i k \mu(\mathbf{r}) \mathbf{H}(\mathbf{r}), \\
& k=\omega / c+i 0 .
\end{aligned}
$$

Here $k$ is the free space wave number. We have added to it an infinitesimal imaginary part to satisfy the boundary conditions at infinity (this will also prove useful for computing various integrals). We have explicitly indicated the dependence of all functions of position on $\mathbf{r}$. However, the frequency $\omega$ is suppressed in the lists of formal arguments of various functions because we focus on just one working frequency. Considering a discrete or continuous superposition of frequencies will naturally lead us to the theory of partially-coherent fields, which is very important in optics but is outside of the scope of this tutorial.

Equations (14) are the closed set of macroscopic Maxwell's equations. If the functions $\epsilon(\mathbf{r}), \mu(\mathbf{r})$ are known and sufficiently "nice" mathematically, we can solve (14) uniquely. On the other hand, if we have a sample of material that is known to be homogeneous internally with some fixed $\epsilon$ and $\mu$, we can perform a set of experimental measurements with quasi-monochromatic radiation that will determine uniquely these quantities at the working frequency. For example, to determine the properties of a homogeneous isotropic slab, one can perform transmission and reflection measurements at several incidence angles, various states of polarization of the incident beam and, perhaps, various widths of the slab. It should be kept in mind that the experiments of this kind are usually carried out with partially-coherent sources of radiation and one should use the appropriate mathematical description of the polarization states of partially-coherent radiation.

Finally, it is often more convenient to consider a given external (incident) field rather than a given external current. This is explained in Sec. 4.B.

\section{THE PHYSICAL MEANING OF POLARIZATION AND MAGNETIZATION}

It is almost universally accepted that the polarization and magnetization fields $\mathbf{P}$ and $\mathbf{M}$ are the differential volume densities of the electric and magnetic dipole moments in continuous media. However, in a typical textbook exposition of the subject, the mathematical arguments in favor of this belief are either missing or flawed. Sometimes it is implied that two functions are point-wise equal if their integrals over a given region are equal [see Eq. (16) below and its discussion]. Sometimes it is remarked that $\mathbf{P}$ and $\mathbf{M}$ can not be determined uniquely from the induced current $\mathbf{J}_{\text {ind }}$ and we must, therefore, apply some additional conditions to define these quantities unambiguously [this argument is discussed in Point (vii) of Sec. 2 above]. In yet other cases, it is argued that $\mathbf{P}$ and $\mathbf{M}$ must have some "physical meaning," and then pictures are drawn of little polarized atoms or current loops. The problem with this approach is that it is relevant only to some very special cases.

The classical interpretation of $\mathbf{P}$ and $\mathbf{M}$, as we will call it, has gained wide acceptance for several reasons. First, it can be viewed as pedagogical, since it gives a visual qualitative explanation of the polarization and magnetization phenomena. Second, the approach seemed to work fine when it could be applied in practice, that is, for molecular gases or simple composites. In other cases, which include pretty much all condensed matter and composites of complex geometry, there was not enough information to say anything. The microscopic charge density in condensed phase could not be computed from simple models.

The situation has changed in 1990-ies with the development of the density-functional theory and related computational methods. When the microscopic charge density was computed in some solids, it became obvious that the classical interpretation does not reproduce the experimental results with an acceptable accuracy. This motivated the development of the modern theory of polarization by King-Smith, Resta and Vanderbilt [9-12]. The main idea of this new theory is that the polarization $\mathbf{P}$ itself (however defined) is not physically important; it is the change of $\mathbf{P}$ that is measurable and can be related to the macroscopic properties of matter. The point of view taken in this tutorial is very similar: the fundamental quantity of interest is the electric current - the time derivative of $\mathbf{P}$ (assuming $\mathbf{M}=0$ ). However, since our discussion is purely classical, we do not touch here the geometric quantum phase, which is central to the modern theory of polarization. We note that recent introductory-level reviews of this theory are available in Refs. [4, 13].

Still, it is hard not to notice that the classical interpretation is surprisingly robust, at least for the electric polarization, and we naturally wish to understand its limits and conditions of ap- 
plicability. Considering the importance of this subject, we will discuss it in detail in this section, primarily, in relation to the electric polarization (magnetization is also discussed briefly). In particular, we will see that the classical interpretation works fine for the Maxwell Garnett theory as long as the characteristic scale of inhomogeneities is much smaller than the wavelength. However, one should be conscious of the conditions of applicability of the classical interpretation, especially when high-frequency phenomena are considered.

\section{A. Polarization and the density of electric dipole moment}

Consider a simple connected region $\mathbb{D}$ of volume $D$ and let the closed and regular surface $S$ be its boundary. The region $\mathbb{D}$ is located arbitrarily with respect to a given sample of continuous medium, which occupies the spatial region $\mathbb{V}$. We wish to compute $\mathbf{d}(t)$ - the macroscopic dipole moment of the matter contained in $\mathbb{D}$. To this end, we can start from Eq. (8) and write

$$
\begin{aligned}
\mathbf{d}(t) & \equiv \int_{\mathbb{D}} \mathbf{r} \rho_{\text {ind }}(\mathbf{r}, t) d^{3} r=-\int_{\mathbb{D}} \mathbf{r}[\nabla \cdot \mathbf{P}(\mathbf{r}, t)] d^{3} r \\
& =\int_{\mathbb{D}} \mathbf{P}(\mathbf{r}, t) d^{3} r+\oint_{\mathbb{S}} \mathbf{r}[\mathbf{P}(\mathbf{r}, t) \cdot d \mathbf{S}] .
\end{aligned}
$$

We have used integration by parts in the second line of (15), $d \mathbf{S}=\hat{\mathbf{n}} d^{2} r$ is the vector element of surface and $\hat{\mathbf{n}}$ is an outward unit normal to $\mathrm{S}$.

If the surface integral in (15) was zero, we could conclude that the classical interpretation is correct. Indeed, we can take $\mathbb{D}$ to be sufficiently small so that $\int_{\mathbb{D}} \mathbf{P}(\mathbf{r}, t) d^{3} r \approx \mathbf{P}\left(\mathbf{r}_{0}\right) D$, where $\mathbf{r}_{0}$ is a point inside $\mathbb{D}$. Then $\mathbf{P}\left(\mathbf{r}_{0}\right) \approx \mathbf{d} / D$ is, indeed, the dipole moment per unit volume. But there is no reason to believe that the surface integral in (15) is zero. An obvious counter-example is a statically polarized piece of metal. Since the macroscopic charge can accumulate in conductors only on the surface (this result holds at arbitrary frequencies if the conductor is internally homogeneous), the dipole moment of any interior volume element is zero while the polarization $\mathbf{P}$ is not. In fact, the macroscopic dipole moment of any internal region of a homogeneous material is zero.

Thus, equation (15) written for the general case does not tell us much. To make a more useful statement, we can take $\mathbb{D} \supset \mathbb{V}$ so that the surface $S$ is in vacuum and completely encloses the object. The surface integral in this case vanishes, and we obtain for the total dipole moment of the object

$$
\mathbf{d}_{\mathrm{tot}}(t)=\int_{\mathbb{V}} \mathbf{P}(\mathbf{r}, t) d^{3} r .
$$

Therefore, the total dipole moment of any electrically neutral object is equal to the integral of $\mathbf{P}$ over its volume. This result is quite general but, of course, it does not mean that $\mathbf{P}$ is the differential density of dipole moment. Indeed, even if the integrals of two functions evaluated over the same region are equal, the functions can still be different.

However, we can use the result (16) constructively if we have a microscopic model or some "more fundamental" expression for the density of charge. As discussed above, this expression can come from one of the theoretical models such as the Lorentz model, the Drude model, the hydrodynamic model; it can result from an $a b$ initio DFT calculation, and so on. Let us say that somehow we have computed a fine-scale density of charge $\varrho$. We expect the following equality to hold:

$$
\int_{\mathbb{V}} \mathbf{P}(\mathbf{r}, t) d^{3} r=\int_{\mathbb{V}} \mathbf{r} \varrho(\mathbf{r}, t) d^{3} r=\mathbf{d}_{\mathrm{tot}} .
$$
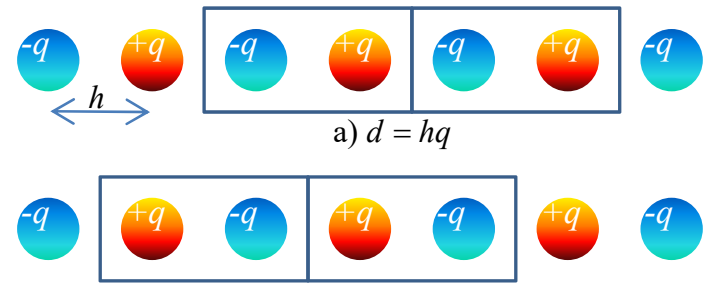

b) $d=-h q$

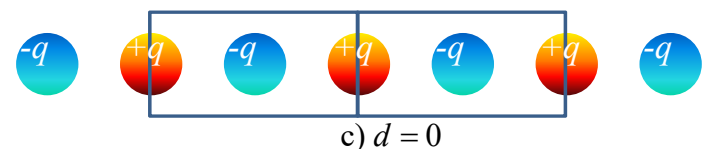

Fig. 1. One-dimensional illustration of the ambiguity of defining the dipole moment of an element of volume. Idea of the figure is borrowed from Ref. [13].

In other words, the total dipole moment of the object should be the same whether we compute it by using the macroscopic polarization function $\mathbf{P}$ or the microscopic charge density $\varrho$. We hope to use this condition to establish a relation between $\mathbf{P}$ and $\varrho$. Here is how we can proceed.

(i) Let us first apply the macroscopic theory to a geometry in which the polarization function inside the object is constant and equal to $\mathbf{P}$. Examples include a sphere, an ellipsoid or a relatively thin slab at a zero or low frequency. Then $\mathbf{d}_{\text {tot }}=V \mathbf{P}$.

(ii) Let us break the object into $N$ elementary cells $\mathbf{C}_{n}$ so that $\mathbb{V}=\cup_{n} \mathbb{C}_{n}$. Let us assume that the function $\varrho(\mathbf{r}, t)$ is periodic. Then the microscopic dipole moment of each cell is $\mathbf{d}_{\mathrm{C}}=\int_{\mathrm{C}} \mathbf{r} \varrho(\mathbf{r}) d^{3} r$.

(iii) From the condition (17), we conclude that $V \mathbf{P}=N \mathbf{d}_{\mathbb{C}}$ and, consequently, $\mathbf{P}=\mathbf{d}_{C} / C$, where $C=V / N$ is the volume of an elementary cell.

We thus have made an identification of $\mathbf{P}$ as the density of microscopic dipole moment in a very special case. In particular, we have assumed that the geometry and frequency are such that the macroscopic polarization is spatially uniform inside the sample, the sample is dividable into completely identical cells and the electromagnetic response of all cells is identical, including the cells at the boundary and in the interior of the sample.

All the conditions described above are important. For example, consider the physical situation sketched in Fig. 1. Here the charge distribution is perfectly periodic. However, if we forget about the left and right ends of the chain, there is no unique definition of the unit cell. We can define the unit cell so that its dipole moment takes any value in the interval $[-q h, q h]$. Therefore, if we want to make a meaningful choice, the condition (ii) must be used. Application of this condition depends on the left and right ends of the chain, which are not shown in the figure. If the medium is electrically neutral, there are only two possible terminations: either the left end is negative and the right end is positive or vice versa. In each case we can break the medium completely into identical elementary cells. In one case, the macroscopic polarization of the medium $P_{z}$ (along the chain) is $+q / h^{2}$ and, in the other case, it is $-q / h^{2}$ (we assume that each cell is cubic); the intermediate values of $P_{z}$ are not possible.

The above example suggests that what happens at the medium boundaries is really important. It is not possible to use a microscopic model of a medium without implicit or explicit consideration of the boundaries. Moreover, the familiar 


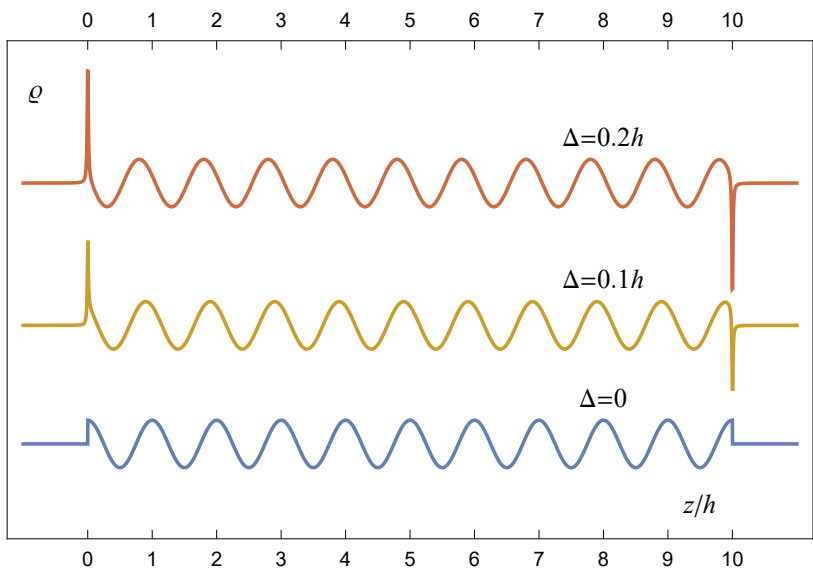

Fig. 2. Illustration of the charge density (18) for $L=10 h$. Here Lorentzians of the width $\gamma=0.01 \mathrm{~h}$ are plotted instead of true delta-functions.

correspondence between $\mathbf{P}$ and the dipole moment of a cell can easily break down if the cells adjacent to the boundaries are in some way not equivalent to the cells in the interior.

Here is a simple one-dimensional example illustrating the importance of boundaries. Consider a microscopic charge distribution $\varrho(z)$ of a sample contained between the planes $z=0$ and $z=L=N h$, which is given by the expression

$$
\begin{aligned}
& \varrho(z)=\varrho_{0}\left\{\cos \left(2 \pi \frac{z+\Delta}{h}\right)\right. \\
& \left.+\frac{h}{2 \pi} \sin \left(2 \pi \frac{\Delta}{h}\right)[\delta(z)-\delta(z-L)]\right\}, 0 \leq z \leq L .
\end{aligned}
$$

This function (18) is obtained by taking the unperturbed charge density $\varrho_{0} \cos (2 \pi z / h)$ in the interval $0 \leq z \leq L$ and "shifting" it to the left by $\Delta$. We assume that there is a hard wall at $z=0$ and the charge can not leak through it. So all positive charge that would otherwise go through the surface is accumulated in the plane $z=0$ and forms a surface charge density. An analogous process occurs at the other boundary $z=L$ except that there the charge of opposite sign is accumulated. We can imagine that the shift $\Delta$ is proportional to the macroscopic field $E_{z}$ inside the medium, say, $\Delta=\left(\chi / \varrho_{0}\right) E_{z}$, where $\chi$ is a proportionality constant. Then the response of the medium is linear as long as $\Delta \ll h$. The function (18) is illustrated in Fig. 2 for different values of $\Delta$.

Let us now break the medium into the elementary cells $n h \leq$ $z<(n+1) h, n=0,1, \ldots, N-1$. It is easy to see that each elementary cell in the interior of the medium (that is, for $1 \leq$ $n \leq N-2)$ acquires a dipole moment per unit surface $d_{C}=$ $\varrho_{0}\left(h^{2} / 2 \pi\right) \sin (2 \pi \Delta / h)$. According to the conventional wisdom, the medium should be assigned the macroscopic polarization

$$
P_{z}=\frac{\varrho_{0} h}{2 \pi} \sin \left(2 \pi \frac{\Delta}{h}\right) \underset{\Delta / h \rightarrow 0}{\longrightarrow} \varrho_{0} \Delta .
$$

Comparing this with $P_{z}=[(\epsilon-1) / 4 \pi] E_{z}$ and $\Delta=\left(\chi / \varrho_{0}\right) E_{z}$, we would conclude that the medium has the permittivity $\epsilon=$ $1+4 \pi \chi$.

But the conventional wisdom is wrong in this case. The problem is that the boundary cells are not equivalent to the cells in the interior. Of course, the boundary cells are not electrically neutral and, therefore, we can not compute unambiguously their dipole moments. However, we can compute the total dipole moment of the whole structure, and it turns out to be equal to zero. It happens so that the dipole moment of the first and last cells (considered together) cancels exactly the dipole moment of all internal cells. If we assign the medium the dielectric permittivity $\epsilon=1+4 \pi \chi$, we would get an obviously incorrect result. In fact, and somewhat paradoxically, the correct value of $\epsilon$ is 1 ; the medium is not macroscopically different from vacuum.

It is interesting to note that the total dipole moment of the sample remains zero if we replace the delta-functions in (18) by Lorentzians of arbitrary width and unit integral weight.

Another way to understand the above result is to consider the microscopic current $j_{z}(z)$. Let us assume that $\Delta$ is a function of time with the derivative $\dot{\Delta}$. Then we can use the continuity equation $\partial \varrho / \partial t+\partial j_{z} / \partial z=0$ to compute the current. The result is $j_{z}=-\varrho_{0} \dot{\Delta} \cos [2 \pi(z+\Delta) / h]$. We have $\int_{0}^{L} j_{z} d z=0$ and, moreover, the average of $j_{z}$ computed over an arbitrarily-defined cell is also zero, viz, $\int_{a}^{a+h} j_{z} d z=0$ for $0 \leq a \leq L-h$.

We have just obtained an illustration of one important fact: unlike the dipole moment, the cell average of the microscopic current is independent of the choice of elementary cell. But this is only natural: polarization and magnetization are introduced as auxiliary quantities while the current is fundamental. Analogously, various multipole moments that are used in the classical interpretation are auxiliary quantities whose introduction can be avoided altogether.

We now need to clarify that the example of Eq. (18) and Fig. 2 illustrates just one particular reason why the classical interpretation can be wrong: the possibility of charge leakage through the cell boundaries. This possibility is the main objection to the classical interpretation that is raised in the modern theory of polarization. We can, however, envisage a physical situation in which every elementary cell contains a localized distribution of charge that can not leak through the cell boundaries. This situation was referred to as the Clausius-Mossotti picture in Ref. [4]. It is well understood now that the Clausius-Mossotti picture is not a realistic model for polarization of solids. Still, the model may seem to be not so bad for the problem of homogenization of macroscopic composite materials. For example, we can consider isolated metallic inclusions in a dielectric host or even in vacuum. In the latter case, the charge can definitely not leak through the cell boundaries.

However, there is another reason why the classical interpretation can fail. According to the condition (ii) above, we should be able to break a macroscopic sample into identical elementary cells. But if the size of a unit cell is not very small compared to the free-space wavelength, such partitioning of the medium is not possible. The fields in two adjacent cells will never be identical in this case. Therefore, the assumption that $\mathbf{d}_{\text {tot }}=N \mathbf{d}_{\mathrm{C}}$ will not hold. One can also say that an elementary cell is not a physically small volume in this case. For the problem of homogenization of macroscopic composites, this objection is much more important than the charge leakage.

We can now make the following conclusions.

(i) There are two main objections to the classical interpretation: charge leakage through the cell boundaries and accumulation of non-negligible phase between two neighboring cells. These objections come to the fore in different physical situations. The first objection can be unimportant for macroscopic composite media while the second objection is almost never important for the theory of molecular polarization of natural materials.

(ii) In some cases, neither of the two objections apply and 
we can safely use the classical interpretation to compute $\epsilon$. In particular, the Maxwell Garnett theory relies on this approach. However, one should keep in mind that the classical interpretation has conditions of applicability.

(iii) To be used constructively, the classical interpretation is not required to be valid for all possible samples. In fact, this is hardly ever possible. For example, even at a very low frequency, we can envisage a sample that is so large that the electromagnetic fields in its elementary cells are not equivalent and the equality $\mathbf{d}_{\text {tot }}=N \mathbf{d}_{\mathrm{C}}$ does not hold. What we actually need is the existence of at least some sample for which all the conditions of applicability of the classical interpretation are met. We can compute $\epsilon$ for this particular sample by using the classical interpretation and then rely on the fact that $\epsilon$ is independent of the overall shape and size of a sample.

\section{B. Magnetization and the density of magnetic dipole moment}

Although we do not discuss magnetic effects in any detail in this tutorial, we will now look briefly at the vector of magnetization M. The main point that we wish to make is that there is no direct analogy between $\mathbf{P}$ and $\mathbf{M}$ and the arguments used above for $\mathbf{P}$ can not be applied to $\mathbf{M}$ without additional restrictions.

The classical interpretation of magnetization is even more problematic because an integral relation of the form (16) does not generally hold for $\mathbf{M}$. Indeed, the macroscopic induced magnetic moment $\mathbf{m}$ of the region $\mathbb{D}$ is by definition

$$
\mathbf{m}=\frac{1}{2 c} \int_{\mathbb{D}} \mathbf{r} \times \mathbf{J}_{\text {ind }} d^{3} r,
$$

where $\mathbf{J}_{\text {ind }}$ is given by (6). We can write $\mathbf{m}=\mathbf{m}_{1}+\mathbf{m}_{2}$, where

$$
\mathbf{m}_{1}=\frac{1}{2 c} \int_{\mathbb{D}} \mathbf{r} \times \frac{\partial \mathbf{P}}{\partial t} d^{3} r, \mathbf{m}_{2}=\frac{1}{2} \int_{\mathbb{D}}(\mathbf{r} \times \nabla \times \mathbf{M}) d^{3} r .
$$

The term $\mathbf{m}_{1}$ contains the electric polarization vector and is usually disregarded at low frequencies. However, at high frequencies, or even in the case when the medium supports a steady current, this term is not zero. To evaluate the second term, we can write identically $\mathbf{r} \times \nabla \times \mathbf{M}=\nabla(\mathbf{r} \cdot \mathbf{M})-\mathbf{M}-(\mathbf{r} \cdot \nabla) \mathbf{M}$. Therefore,

$$
\mathbf{m}_{2}=\frac{1}{2}\left[\oint_{S}(\mathbf{r} \cdot \mathbf{M}) d \mathbf{S}-\int_{\mathbb{D}} \mathbf{M} d^{3} r-\int_{\mathbb{D}}(\mathbf{r} \cdot \nabla) \mathbf{M} d^{3} r\right] .
$$

The last integral can be evaluated by parts as

$$
\int_{\mathbb{D}}(\mathbf{r} \cdot \nabla) \mathbf{M} d^{3} r=\oint_{S} \mathbf{M}(\mathbf{r} \cdot d \mathbf{S})-3 \int_{\mathbb{D}} \mathbf{M} d^{3} r .
$$

Collecting everything together, we obtain after some additional re-arrangement

$$
\mathbf{m}_{2}=\int_{\mathbb{D}} \mathbf{M} d^{3} r-\frac{1}{2} \oint_{S} \mathbf{r} \times \mathbf{M} \times d \mathbf{S} .
$$

Again, we see that there is a surface term, which disappears only if $\mathrm{S}$ is drawn outside of the body.

Let us now take $\mathbb{D} \supset \mathbb{V}$ so that the surface integral vanishes. Then the total magnetic dipole moment of the object is

$$
\mathbf{m}_{\mathrm{tot}}=\int_{\mathbb{V}} \mathbf{M} d^{3} r+\frac{1}{2 c} \int_{\mathbb{V}} \mathbf{r} \times \frac{\partial \mathbf{P}}{\partial t} d^{3} r .
$$

This equation is more complicated than the analogous result for $\mathbf{d}_{\text {tot }}$ (16). We can use the arguments of previous subsection to establish a mathematical connection between the magnetic moment of an elementary cell $\mathbf{m}_{\mathbb{C}}$ and the macroscopic magnetization $\mathbf{M}$ only if the second term in the right-hand side of (25) is negligibly small, which can be the case at sufficiently low frequencies. Moreover, the transformation of $\mathbf{m}_{\text {tot }}$ under a coordinate shift $\mathbf{r} \rightarrow \mathbf{r}+\mathbf{a}$ is given by

$$
\mathbf{m}_{\text {tot }} \rightarrow \mathbf{m}_{\text {tot }}+\frac{1}{2 c} \mathbf{a} \times \frac{\partial}{\partial t} \int_{\mathbb{V}} \mathbf{P} d^{3} r=\mathbf{m}_{\text {tot }}+\frac{1}{2 c} \mathbf{a} \times \frac{\partial \mathbf{d}_{\mathrm{tot}}}{\partial t} .
$$

It can be seen that $\mathbf{m}_{\text {tot }}$ is defined unambiguously only if the term proportional to $\partial \mathbf{d}_{\mathrm{tot}} / \partial t$ is negligibly small.

The above discussion concerns magnetization caused by the electric currents, which are often referred to as orbital, although they can also include the conductivity current. Of course, the orbital currents obey quantum laws at the microscopic level. However, macroscopically, they can be viewed as classical. Further details of the modern theory of orbital magnetization can be found in [14, 15]. In contrast, ferromagnetism is a macroscopic quantum effect, which can not be fully understood within the theoretical framework of classical physics. Nevertheless, the classical interpretation of $\mathbf{M}$ works fine for ferromagnetism. This is so, firstly, because the last term in (26) is negligible at the low frequencies for which the ferromagnetic effect is non-negligible and, secondly, because the elementary Amperian current loops (the quantum-mechanical expectations of the electric current associated with an electron spin) fit well into the Clausius-Mossotti picture outlined above. In other words, the conditions of applicability of the classical interpretation that were discussed above for the case of electric polarization also hold for the elementary current loops in ferromagnetics.

\section{WAVES ON LATTICES OF POLARIZED POINT PARTI- CLES}

In the first part of this tutorial, we have derived the Maxwell Garnett approximation for a collection of point noninteracting dipoles at zero frequency. We now wish to construct a more general theory. Specifically, we will consider arbitrary frequencies and allow the dipoles to interact. We will, however, restrict attention to a very simple geometry: point-like, sphericallysymmetric particles arranged on a cubic lattice. We will show that the Maxwell Garnett mixing formula follows from the dispersion relation for the electromagnetic waves propagating on such a lattice in the long wavelength limit. We will not use the classical interpretation of polarization as the dipole moment per unit volume for arriving at this conclusion. However, the classical interpretation is valid in the long wavelength limit and can be used to derive the same result.

The model of electromagnetically-interacting point-like polarizable particles arranged on a three-dimensional lattice possesses an intuitive physical appeal. Historically, many authors have considered this model [16-21]. Most if not all of these references assume that the particles are embedded in vacuum. We will make an unfortunate compromise and work under the same assumption here, although it could have been more logical to start with the model of small inclusions in a host of permittivity $\epsilon_{h} \neq 1$. The main reason for our choice is simplicity. Introduction of a host medium is not conceptually difficult but will shift attention away from the key points, which we wish to avoid. However, we hope that anyone who has followed through the derivations of this section will be able to repeat them for a more general host. Alternatively, the end result of such a calculation can be obtained from the less general result 
of this section by the substitution $\epsilon_{\text {eff }} \rightarrow \epsilon_{\text {eff }} / \epsilon_{h}, \epsilon_{i} \rightarrow \epsilon_{i} / \epsilon_{h}$, where $\epsilon_{\text {eff }}$ is the effective permittivity of the structure and $\epsilon_{i}$ is the permittivity of inclusions. Towards the end of this section, we will apply this transformation to derive the Maxwell Garnett approximation for a general two-component mixture.

\section{A. Polarizability of a small particle beyond the static limit}

Consider a small particle illuminated by an external monochromatic field of the complex amplitude $\mathbf{E}(\mathbf{r})$ and frequency $\omega$. If the particle size $a$ is much smaller than the wavelength of the incident radiation, it can be characterized by the dipole polarizability tensor $\hat{\alpha}$, which is defined as the linear coefficient in the expression $\mathbf{d}=\hat{\alpha} \mathbf{E}\left(\mathbf{r}_{0}\right)$. Here $\mathbf{d}$ is the complex amplitude of the dipole moment and $\mathbf{r}_{0}$ is a point inside the particle. If the particle has the cubic symmetry, we can write $\hat{\alpha}=\alpha \hat{I}$, where $\alpha$ is a scalar and $\hat{I}$ is the identity tensor. For simplicity, we will consider the scalar case in this section. However, the foregoing discussion also applies to all principal components of the tensor $\hat{\alpha}$, assuming it can be diagonalized by an orthogonal transformation.

The polarizability is not arbitrary but satisfies certain constraints. A well known result is that the extinction cross section of the particle (if excited by a plane wave) is $\sigma_{e}=4 \pi k \operatorname{Im} \alpha$. Since $\sigma_{e}>0$, we conclude that $\operatorname{Im} \alpha>0$. But this is not the most interesting constraint. Another inequality can be obtained by noting that the scattering cross section under the same excitation conditions is $\sigma_{s}=(8 \pi / 3) k^{4}|\alpha|^{2}$. We know that $\sigma_{e} \geq \sigma_{s}$, where the equality holds for an ideally non-absorbing particle. From this, we find that

$$
\operatorname{Im}(1 / \alpha) \leq-2 k^{3} / 3
$$

The above result may seem strange if we recall the quasistatic formula for the polarizability of a sphere (FP3). This formula yields a real-valued result if $\epsilon$ is real at the working frequency. Even if we allow $\epsilon$ to have a small imaginary part to satisfy the condition $\operatorname{Im} \alpha>0$, the inequality (27) might still not hold.

The apparent contradiction can be resolved by noting that (FP3) is not quite accurate at finite frequencies: it does not account for the leading-order radiative correction (to the inverse polarizability), which is equal to $-2 i k^{3} / 3$. This correction can be relatively very small. However, it is needed to satisfy the optical theorem. When the electromagnetic interaction of several small particles is considered, not accounting for the radiative correction can yield strange results that contradict energy conservation $[22,23]$.

It turns out that corrections to $\operatorname{Re}(1 / \alpha)$ also exist and are even of lower order in $k$. We can refer to these corrections as to non-radiative since they are not associated with scattering. Unfortunately, these corrections are not uniquely defined. Different authors give different expressions for the non-radiative corrections [24]. The reason for this ambiguity is an irremovable singularity of the Green's tensor for the Maxwell's equations; we will encounter this singularity momentarily. As a result, the non-radiative corrections depend not only on the particle shape (which could be expected) but also on the form of the incident field and on the reference point $\mathbf{r}_{0}$.

On the other hand, the first non-vanishing radiative correction is universal. This is so because the imaginary part of the Green's tensor does not experience the singularity mentioned above. One can argue that it is the correction to $\operatorname{Im}(1 / \alpha)$ that is physically important and should be retained even in the limit $k a \rightarrow 0$. In accord with this argument, we will decompose the inverse polarizability as

$$
1 / \alpha=1 / \tilde{\alpha}-2 i k^{3} / 3
$$

where $\tilde{\alpha}$ is the polarizability with all the corrections up to the order of $O\left(k^{2}\right)$. We do not really know the exact form of these corrections without formulating the problem more precisely. All we can say for sure is that $\tilde{\alpha} \rightarrow \alpha_{\mathrm{LL}}$ when $k a \rightarrow 0$, where $\alpha_{\mathrm{LL}}$ is the quasistatic "Lorentz-Lorenz" polarizability of the particle. For example, $\alpha_{\mathrm{LL}}$ is given by (FP3) for a sphere or by (FP32) for an ellipsoid. The difference $\alpha_{L L}-\tilde{\alpha}$ is of the order of $O\left(k^{2}\right)$ if the particle has a center of symmetry and of the order of $O(k)$ otherwise. Note that $\tilde{\alpha}$ can be complex or real (in non-absorbing particles).

One last question that we need to address is the following. If we account for the effects that are of the order of $O\left(k^{2}\right)$, is it correct to neglect all higher-order multipole moments? Strictly speaking, the answer is "no". The magnetic dipole and electric quadrupole moments of small particles are quantities of the order of $O\left(k^{2}\right)$, same as the difference $1 / \tilde{\alpha}-1 / \alpha_{\mathrm{LL}}$. We therefore will work in the regime when the non-radiative corrections and the higher-order multipole moments can be neglected.

As for the radiative correction, we will see that, in the particular problem we are solving, it exactly cancels. The cancellation takes place because a plane wave can propagate on an infinite, perfectly ordered lattice without scattering. Formally, the same result could be obtained if we simply forgot about the radiative correction and neglected the terms that are of the order of $O\left(k^{2}\right)$ and higher in all formulas. It may seem therefore that our discussion of the radiative correction was redundant. But this is not truly so. In problems that actually involve scattering, e.g., for finite or disordered sets of dipoles, accounting for the radiative correction can be crucial; its neglect can result in the appearance of non-physical resonances or divergences and, ultimately, in violations of energy conservation. On the other hand, neglect of the non-radiative corrections does not lead to anything dramatic or unphysical. One can also say that $\operatorname{Re}(1 / \alpha)$ and $\operatorname{Im}(1 / \alpha)$ are responsible for different physical effects and should be considered separately.

\section{B. Green's tensor beyond the static limit}

We next need an expression for the electric field radiated by a point dipole. This result is given by the free-space Green's tensor for the Maxwell's equations. We have already encountered the Green's tensor in the static limit in the first part of this tutorial [see (FP8)]. Now we need to generalize this result to finite frequencies.

We start from the frequency domain macroscopic Maxwell's equations (14). We consider a non-magnetic medium with $\mu=$ 1 and let $\epsilon(\mathbf{r})=1+4 \pi \chi(\mathbf{r})$, where the susceptibility $\chi(\mathbf{r})$ is zero in vacuum. Upon substitution of $\mathbf{H}$ from the second into the first equation of (14), and by using the definitions (10),(12) to write $\chi(\mathbf{r}) \mathbf{E}(\mathbf{r})=\mathbf{P}(\mathbf{r})$, we obtain

$$
\left[(\nabla \times \nabla \times)-k^{2}\right] \mathbf{E}(\mathbf{r})=4 \pi k^{2} \mathbf{P}(\mathbf{r})+\frac{4 \pi i k}{c} \mathbf{J}_{\operatorname{ext}}(\mathbf{r}) .
$$

From linearity, we can write the solution to the above equation in the following form:

$$
\mathbf{E}(\mathbf{r})=\mathbf{E}_{\text {ext }}(\mathbf{r})+\int \hat{G}\left(\mathbf{r}, \mathbf{r}^{\prime}\right) \mathbf{P}\left(\mathbf{r}^{\prime}\right) d^{3} r^{\prime}
$$


where the field $\mathbf{E}_{\text {ext }}$ and the Greens tensor $\hat{G}$ satisfy

$$
\begin{aligned}
& {\left[(\nabla \times \nabla \times)-k^{2}\right] \mathbf{E}_{\mathrm{ext}}(\mathbf{r})=\frac{4 \pi i k}{c} \mathbf{J}_{\mathrm{ext}}(\mathbf{r}),} \\
& {\left[(\nabla \times \nabla \times)-k^{2}\right] \hat{G}\left(\mathbf{r}, \mathbf{r}^{\prime}\right)=4 \pi k^{2} \hat{I} \delta\left(\mathbf{r}-\mathbf{r}^{\prime}\right),}
\end{aligned}
$$

and $\hat{I}$ is the identity tensor.

We will refer to the solution of (31a), $\mathbf{E}_{\text {ext }}$, as to the external or incident field. Here $\mathbf{E}_{\text {ext }}$ is the field that would have existed everywhere in space in the absence of the sample. Note that

$$
\mathbf{E}_{\text {ext }}(\mathbf{r})=-\frac{1}{i \omega} \int \hat{G}\left(\mathbf{r}, \mathbf{r}^{\prime}\right) \mathbf{J}_{\mathrm{ext}}\left(\mathbf{r}^{\prime}\right) d^{3} r^{\prime}
$$

Electromagnetic problems are typically formulated in terms of a known external field rather than external current. Equation (30) is one possible mathematical formulation of this approach.

In contrast, the second term in (30) is the field that is generated, or scattered by the sample; we denote this term by $\mathbf{E}_{\text {scatt, }}$ so that

$$
\mathbf{E}_{\text {scatt }}(\mathbf{r})=\int \hat{G}\left(\mathbf{r}, \mathbf{r}^{\prime}\right) \mathbf{P}\left(\mathbf{r}^{\prime}\right) d^{3} r^{\prime}
$$

The physical significance of the Green's tensor can be understood as follows. Consider scattering of an external field by a small particle occupying the region $\mathbb{V}$ whose characteristic size $a$ is much smaller than the free space wavelength so that $k a \ll 1$. Let the point $\mathbf{r}_{0} \in \mathbb{V}$ be the "center" of $\mathbb{V}$. Then, for $\left|\mathbf{r}-\mathbf{r}_{0}\right| \gg a$, we can write

$$
\mathbf{E}_{\text {scatt }}(\mathbf{r}) \approx \hat{G}\left(\mathbf{r}, \mathbf{r}_{0}\right) \int_{\mathbb{V}} \mathbf{P}(\mathbf{r}) d^{3} r=\hat{G}\left(\mathbf{r}, \mathbf{r}_{0}\right) \mathbf{d},
$$

where we have used (16) to obtain the second equality. Therefore, the Green's tensor $\hat{G}\left(\mathbf{r}, \mathbf{r}_{0}\right)$ gives the radiation field at point $\mathbf{r}$ of an elementary dipole at point $\mathbf{r}_{0}$.

We will now use (31b) to compute $\hat{G}$. Accounting for the translational invariance of free space, we can write

$$
\hat{G}\left(\mathbf{r}, \mathbf{r}^{\prime}\right)=\hat{F}\left(\mathbf{r}-\mathbf{r}^{\prime}\right)=\int \hat{K}(\mathbf{p}) e^{i \mathbf{p} \cdot\left(\mathbf{r}-\mathbf{r}^{\prime}\right)} \frac{d^{3} p}{(2 \pi)^{3}} .
$$

Substitution of this ansatz and the similar Fourier representation of the delta-function $\delta\left(\mathbf{r}-\mathbf{r}^{\prime}\right)$ into (31b) results in the equation

$$
\left[(\mathbf{p} \times \mathbf{p} \times)+k^{2}\right] \hat{K}(\mathbf{p})=-4 \pi k^{2} \hat{I} .
$$

This is a $3 \times 3$ matrix equation for $\hat{K}(\mathbf{p})$. We can use the identity $(\mathbf{p} \times \mathbf{p} \times)=\mathbf{p} \otimes \mathbf{p}-p^{2} \hat{I}$ to facilitate its solution. The result is

$$
\hat{K}(\mathbf{p})=4 \pi \frac{k^{2} \hat{I}-\mathbf{p} \otimes \mathbf{p}}{p^{2}-k^{2}} .
$$

Here $\otimes$ denotes tensor product of two vectors.

The real-space Green's tensor can be obtained by computing the Fourier integral (35). To this end, we can replace the tensor $\mathbf{p} \otimes \mathbf{p}$ by $\nabla_{\mathbf{r}} \otimes \nabla_{\mathbf{r}^{\prime}}$. Then we can perform the angular integration by using the elementary integration rules and the ingratiation over $p$ by residues. We can use the symmetry of the integrand to extend integration to the whole real axis; also, we should keep in mind that $k$ has an infinitesimal imaginary part according to (14c). The result of this calculation is

$$
\hat{G}\left(\mathbf{r}, \mathbf{r}^{\prime}\right)=\left(k^{2} \hat{I}-\nabla_{\mathbf{r}} \otimes \nabla_{\mathbf{r}^{\prime}}\right) \frac{\exp \left(i k\left|\mathbf{r}-\mathbf{r}^{\prime}\right|\right)}{\left|\mathbf{r}-\mathbf{r}^{\prime}\right|},
$$

or

$$
\hat{F}(\mathbf{r})=\left(k^{2} \hat{I}+\nabla \otimes \nabla\right) \frac{\exp (i k r)}{r} .
$$

Note that the signs in (38),(39) are correct. Similarly to the static case, (38) is singular due to the differentiation of the nonanalytical expression $|\mathbf{r}|$. We can write

$$
\hat{F}(\mathbf{r})=-\frac{4 \pi}{3} \delta(\mathbf{r})+\hat{F}_{R}(\mathbf{r}),
$$

where $\hat{F}_{R}$ is "regular" in the sense that

$$
\int_{|\mathbf{r}|<a} \hat{F}_{R}(\mathbf{r}) d^{3} r \underset{a \rightarrow 0}{\longrightarrow} 0 .
$$

We have written the word "regular" in quotes because the property (41) depends on the integration region and $\hat{F}_{R}$ is not truly regular; it still contains a singularity. As a result, some physically-important integrals involving $\hat{F}_{R}$ are conditionally convergent. Indeed, by performing the differentiation carefully everywhere except for $\mathbf{r}=0$, we obtain the following result:

$$
\hat{F}_{R}(\mathbf{r})=\left[\left(\frac{k^{2}}{r}+\frac{i k}{r^{2}}-\frac{1}{r^{3}}\right) \hat{I}+\left(-\frac{k^{2}}{r}-\frac{3 i k}{r^{2}}+\frac{3}{r^{3}}\right) \frac{\mathbf{r} \otimes \mathbf{r}}{r^{2}}\right] e^{i k r} .
$$

We thus see that $\hat{F}_{R}(\mathbf{r})$ diverges as $r^{-3}$ when $\mathbf{r} \rightarrow 0$. However, the real and imaginary parts of $\hat{F}_{R}$ behave differently. The expansion of $\hat{F}(\mathbf{r})$ in powers of $r$ near $r=0$ is of the form

$$
\begin{aligned}
& \operatorname{Re} \hat{F}_{R}(\mathbf{r})=\left(-\frac{1}{r^{3}}+\frac{k^{2}}{2 r}\right) \hat{I}+\left(\frac{3}{r^{3}}+\frac{k^{2}}{2 r}\right) \frac{\mathbf{r} \otimes \mathbf{r}}{r^{2}}+O(r), \\
& \operatorname{Im} \hat{F}_{R}(\mathbf{r})=\left(\frac{2 k^{3}}{3}-\frac{2 k^{5} r^{2}}{15}\right) \hat{I}+\frac{k^{5} r^{2}}{15} \frac{\mathbf{r} \otimes \mathbf{r}}{r^{2}}+O\left(r^{4}\right) .
\end{aligned}
$$

It can be seen that $\operatorname{Im} \hat{F}_{R}(\mathbf{r})$ has no singularity. This is why the first non-vanishing radiative correction is universal; in fact, it is given by the first term in the right-hand side of (43b).

Let us now evaluate the integral of $\hat{F}_{R}(\mathbf{r})$ over a small ball of radius $a$. We can use the identity $\int_{4 \pi}(\mathbf{r} \otimes \mathbf{r}) d \Omega=\frac{4 \pi}{3} r^{2} \hat{I}$, where $d \Omega$ is the element of solid angle, to compute the angular integrals. We then obtain

$$
\begin{aligned}
\int_{|\mathbf{r}|<a} \hat{F}_{R}(\mathbf{r}) d^{3} r & =\frac{8 \pi}{3} \hat{I}\left[(1-i k a) e^{i k a}-1\right] \\
& \underset{k a \rightarrow 0}{\longrightarrow} \frac{4 \pi}{3} \hat{I}\left[(k a)^{2}+i \frac{2(k a)^{3}}{3}+\ldots\right] .
\end{aligned}
$$

This result is in agreement with (41).

On the other hand, if we evaluate the integral in (44) over a more general nonspherical region, the term $O\left(r^{-3}\right)$ would not integrate to zero. We will observe a physical manifestation of this fact when we consider waves on lattices of point polarizable particles. We have also seen a similar difficulty when we considered anisotropy in the first part of this tutorial (in section 4). All we can say now is that the "regularizing" decomposition (40) is appropriate when spherical particles and regions are considered.

We can also define the "regular" part of the Green's tensor in the spatial Fourier domain. That is, we can write

$$
\hat{K}(\mathbf{p})=-(4 \pi / 3) \hat{I}+\hat{K}_{R}(\mathbf{p}),
$$

where $\hat{F}_{R}$ and $\hat{K}_{R}$ are spatial Fourier transforms of each other. We can easily find that

$$
\hat{K}_{R}(\mathbf{p})=\frac{4 \pi}{3} \frac{\left(2 k^{2}+p^{2}\right) \hat{I}-3 \mathbf{p} \otimes \mathbf{p}}{p^{2}-k^{2}} .
$$

This result will be used in the calculations below. 


\section{Coupled-dipole equations on an infinite lattice}

Consider an assembly of identical, point-like particles of scalar polarizability $\alpha$ located at the positions $\mathbf{r}_{n}$. Let the whole system be illuminated by the external field $\mathbf{E}_{\text {ext }}(\mathbf{r})$. Then the dipole moments of the particles $\mathbf{d}_{n}$ are coupled to the external field and to each other by the coupled-dipole equation

$$
\mathbf{d}_{n}=\alpha\left[\mathbf{E}_{\mathrm{ext}}\left(\mathbf{r}_{n}\right)+\sum_{m \neq n} \hat{G}_{R}\left(\mathbf{r}_{n}, \mathbf{r}_{m}\right) \mathbf{d}_{m}\right]
$$

Note that the term with $m=n$ is left out of the summation in the right-hand side of (47). This reflects our idea that the electric field acting on the $n$-th particle is a superposition of the external field $\mathbf{E}_{\text {ext }}\left(\mathbf{r}_{n}\right)$ and the fields scattered by all particles except for the $n$-th particle itself.

Unfortunately, our exposition of the coupled-dipole equation is by necessity brief. We note only that it is important to use the correct definitions for all quantities appearing in (47). In particular, we should use the polarizability $\alpha$ as it was defined in section 4.A above and not the "bare polarizability" [25] or the quasistatic polarizability $\alpha_{\mathrm{LL}}$. Also, we have used the "regular" part of the Green's tensor, $\hat{G}_{R}$, in (47) rather than $\hat{G}$. In the real-space representation, this is unimportant as long as the $m=n$ term is not included in the summation. However, upon spatial Fourier transformation, the difference will show up. Additional details about the coupled-dipole equations can be found in Refs. [19, 24, 26].

Our goal is to find an eigen-solution to (47) on an infinite lattice and to analyze the corresponding dispersion relation. To this end, we assume that $\mathbf{r}_{n}$ are the vertices of an infinite cubic lattice of pitch $h$, set the external field $\mathbf{E}_{\text {ext }}$ to zero and seek the solution to (47) in the form $\mathbf{d}_{n}=\mathbf{d} \exp \left(i \mathbf{q} \cdot \mathbf{r}_{n}\right)$. Upon substitution of this ansatz into (47), we obtain the equation

$$
\frac{1}{\alpha} \mathbf{d}=\hat{S}(\mathbf{q}) \mathbf{d}
$$

where

$$
\hat{S}(\mathbf{q})=\sum_{\mathbf{r}_{n} \neq 0} \hat{F}_{R}\left(\mathbf{r}_{n}\right) e^{-i \mathbf{q} \cdot \mathbf{r}_{n}}
$$

is the lattice sum, sometimes also referred to as the dipole sum.

Lattice sums are ubiquitous in physics [27] but not always convergent. Correspondingly, a lot of effort has been devoted to computing and regularizing diverging lattice sums. Two approaches to this task are common: allowing the point particles to have higher-order multipole moments or allowing the particles to have a finite size and shape. Ultimately, the two approaches are equivalent since they describe the same physical reality. We will resort to the second approach - that is, we will assume that the particles are small spheres.

We now proceed with the calculation. First, we use the Fourier representation (35) (applied to $\hat{G}_{R}$ ) to re-write (49) as

$$
\hat{S}(\mathbf{q})=\int \frac{d^{3} p}{(2 \pi)^{3}} \hat{K}_{R}(\mathbf{p}) \sum_{\mathbf{r}_{n} \neq 0} e^{i(\mathbf{p}-\mathbf{q}) \cdot \mathbf{r}_{n}} .
$$

To evaluate this expression, we need to complete the summation. This can be accomplished by adding and subtracting unity to the series, which leads us to the expression

$$
\hat{S}(\mathbf{q})=\frac{1}{h^{3}} \sum_{n} \hat{K}_{R}\left(\mathbf{q}+\mathbf{g}_{n}\right)-\int \frac{d^{3} p}{(2 \pi)^{3}} \hat{K}_{R}(\mathbf{p}),
$$

where we have used the Poisson summation formula

$$
\sum_{n} e^{i \mathbf{p} \cdot \mathbf{r}_{n}}=\left(\frac{2 \pi}{h}\right)^{3} \sum_{n} \delta\left(\mathbf{p}-\mathbf{g}_{n}\right), \mathbf{g}_{n}=\frac{2 \pi}{h}\left(n_{x}, n_{y}, n_{z}\right) .
$$

Here $\mathbf{g}_{n}$ are the reciprocal lattice vectors and we can view $n=$ $\left(n_{x}, n_{y}, n_{z}\right)$ as a composite index.

As expected, the expression (51) is divergent: the last term in the right-hand side is just $\hat{F}_{R}(0)$. But $\hat{F}_{R}(0)$ is not well defined. So we will use the regularizing substitution

$$
\begin{aligned}
\int \frac{d^{3} p}{(2 \pi)^{3}} \hat{K}_{R}(\mathbf{p})=\hat{F}_{R}(0) & \longrightarrow \frac{3}{4 \pi a^{3}} \int_{|\mathbf{r}|<a} \hat{F}_{R}(\mathbf{r}) d^{3} r \\
& \approx\left(\frac{k^{2}}{a}+i \frac{2 a^{3}}{3}\right) \hat{I}
\end{aligned}
$$

where we have used the result (44). As mentioned above, this regularization is appropriate in the case when the particles are small spheres of radius $a$.

We now put everything together. We substitute the result (53) into (51) and (51) into (48), use the decomposition (28) for $1 / \alpha$, and obtain

$$
\left(\frac{1}{\tilde{\alpha}}+\frac{k^{2}}{a}\right) \mathbf{d}=\frac{1}{h^{3}} \sum_{n} K_{R}\left(\mathbf{q}+\mathbf{g}_{n}\right) \mathbf{d} .
$$

As expected, the radiative correction has canceled.

\section{Long wavelength approximation}

The dispersion relation (54) is still too complicated for analysis. To proceed, we will need to make the long wavelength approximation. Namely, we will assume that $k h \ll 1$ and $\left|q_{p}\right| h \ll 1$, where $p=x, y, z$. We should keep in mind that, in our model, $a \ll h$, so that $k a \ll 1$ also holds. Then, with the precision of the approximation used, we can write $1 / \tilde{\alpha}+k^{2} / a \approx 1 / \alpha_{\mathrm{LL}}$, where $\alpha_{\mathrm{LL}}$ is the quasistatic polarizability of a particle.

We thus have simplified the left-hand side of (54). In the right-hand side, we will write the term with $\mathbf{g}_{n}=0$ and the rest of the sum separately. We will thus obtain

$$
\frac{1}{\alpha_{\mathrm{LL}}} \mathbf{d}=\frac{1}{h^{3}} \hat{K}_{R}(\mathbf{q}) \mathbf{d}+\frac{1}{h^{3}} \sum_{\mathbf{g}_{n} \neq 0} \hat{K}_{R}\left(\mathbf{q}+\mathbf{g}_{n}\right) \mathbf{d} .
$$

We will now show that the last term in the right-hand side of (55) can be neglected in the long wavelength approximation. Indeed, for $\mathbf{g}_{n} \neq 0$, we can write

$$
\hat{K}_{R}\left(\mathbf{q}+\mathbf{g}_{n}\right) \underset{k h,\left|q_{p}\right| h \rightarrow 0}{\longrightarrow} \frac{4 \pi}{3} \hat{Q}_{n}
$$

where

$$
\hat{Q}_{n}=\hat{I}-3 \frac{\mathbf{g}_{n} \otimes \mathbf{g}_{n}}{g_{n}^{2}}, \mathbf{g}_{n} \neq 0 .
$$

Now, if the particles are arranged on a cubic lattice, the reciprocal lattice is also cubic. We can define a discrete cubic box with excluded center, $\mathbb{B}(L)$, as the set of all reciprocal lattice vectors with the indexes $n=\left(n_{x}, n_{y}, n_{z}\right)$ such that $-L \leq n_{x}, n_{y}, n_{z} \leq L$ except for $n=(0,0,0)$. Then

$$
\sum_{\mathbf{g}_{n} \neq 0} \hat{Q}_{n}=\lim _{L \rightarrow \infty} \sum_{\mathbf{g}_{n} \in \mathbb{B}(L)} \hat{Q}_{n}
$$

But each individual sum $\sum_{\mathbf{g}_{n} \in \mathbb{B}(L)} \hat{Q}_{n}$ is zero. This can be proved by noting that the sum over the surface of each box is 
zero and then representing a box of size $L$ as a set of concentric surfaces with the sizes $L^{\prime}=1,2, \ldots, L$. From this, we conclude that the limit in (58) is also zero and the second term in the right-hand side of (55) can be neglected in the long wavelength approximation.

We now use the above fact and the expression (46) for $\hat{\mathbf{K}}_{R}(\mathbf{q})$ to obtain the following equation:

$$
\frac{1}{\alpha_{\mathrm{LL}}} \mathbf{d}=\frac{4 \pi}{3 h^{3}} \frac{\left(2 k^{2}+q^{2}\right) \hat{I}-3 \mathbf{q} \otimes \mathbf{q}}{q^{2}-k^{2}} \mathbf{d} .
$$

This is the dispersion relation for electromagnetic waves propagating on a cubic lattice of small, spherical, polarizable particles in the long wavelength approximation.

The only thing left for us to do is to consider the polarization states. The effective permittivity of a cubic lattice of small spherical particles can not be anisotropic (from symmetry, the principal axes of the tensor $\hat{\epsilon}_{\text {eff }}$ must coincide with the crystallographic axes, and the directions along all three crystallographic axes are equivalent). Therefore, we must assume that the medium is characterizable by a scalar effective permittivity $\epsilon_{\text {eff }}$ and $q^{2}=k^{2} \epsilon_{\text {eff }}$ (we have assumed here that $\mu_{\text {eff }}=1$, more on this later). Under the same conditions, the medium can support transverse waves for which $\mathbf{q} \cdot \mathbf{d}=0$. Then it follows from (59) that the dispersion equation is

$$
\frac{v}{\alpha_{\mathrm{LL}}}=\frac{4 \pi}{3} \frac{\epsilon_{\mathrm{eff}}+2}{\epsilon_{\mathrm{eff}}-1},
$$

where we have introduced the specific volume per one particle $v=h^{3}$. Note that longitudinal waves can also propagate on the lattice but only if the special condition $h^{3} / \alpha=-8 \pi / 3$ holds (the structure is similar in this case to a non-dissipative plasma). Usually this condition does not hold and we will ignore the longitudinal waves.

Equation (60) is the inverted Clausius-Mossotti relation [compare to (FP16)]. We therefore have derived the ClausiusMossotti relation as the long wavelength limit of the dispersion relation for electromagnetic waves on a cubic lattice of small spherical particles.

The Maxwell Garnett approximation can be obtained if we use a specific expression for $\alpha_{L L}$. Since we have assumed that the particles are small spheres, we will use (FP3). Substituting everything into (60) and inverting the equation, we obtain

$$
\frac{\epsilon_{\mathrm{eff}}-1}{\epsilon_{\mathrm{eff}}+2}=f \frac{\epsilon-1}{\epsilon+2}
$$

where $f=4 \pi a^{3} / 3 v$ is the volume fraction of inclusions. This is the Maxwell Garnett mixing formula for inclusions of the permittivity $\epsilon$ in vacuum. We can generalize this result to inclusions of the permittivity $\epsilon_{i}$ in a host medium of the permittivity $\epsilon_{h}$ by using the substitution $\epsilon_{\text {eff }} \rightarrow \epsilon_{\text {eff }} / \epsilon_{h}$ and $\epsilon \rightarrow \epsilon_{i} / \epsilon_{h}$. We thus obtain

$$
\frac{\epsilon_{\mathrm{eff}}-\epsilon_{h}}{\epsilon_{\mathrm{eff}}+2 \epsilon_{h}}=f \frac{\epsilon_{i}-\epsilon_{h}}{\epsilon_{i}+2 \epsilon_{h}} .
$$

This is the Maxwell Garnett mixing formula written in the form (FP25).

\section{E. Beyond the long wavelength approximation}

We now discuss briefly what can happen beyond the long wavelength approximation. The material of this subsection is not a self-contained exposition but a collection of a few basic ideas and leads for further reading and exploration.
Recall that we have neglected the non-radiative corrections to the inverse polarizability and replaced the term $1 / \tilde{\alpha}+k^{2} / a$ by $1 / \alpha_{\mathrm{LL}}$. We can not make a more precise statement about the inverse polarizability without abandoning the model of pointlike dipoles. However, we can still use this model and introduce physically-meaningful corrections to the long wavelength approximation if $a \ll h$. In this case, the inequality $k a \ll 1$ can hold quite strongly, but the inequalities $k h \ll 1$ and $\left|q_{p}\right| h \ll 1$ can be not so strong. We then expand the term $K_{R}\left(\mathbf{q}+\mathbf{g}_{n}\right)$ with $\mathbf{g}_{n} \neq 0$ [in (55)] in powers of $k$ and $\mathbf{q}$. The long-wavelength result (56) will give the zeroth order in this expansion. Some of the expansion terms will sum to zero due to symmetry but others will remain. If we make all expressions dimensionless by multiplying the numerator and denominator in the expression for $K_{R}\left(\mathbf{q}+\mathbf{g}_{n}\right)$ by $h$, the corrections will be of the order of $O\left((k h)^{n}\right)$ and $O\left(\left(q_{p} h\right)^{n}\right)$, where $n$ is even. This is true for all systems with a center of symmetry.

The terms proportional to $(k h)^{2},(k h)^{4}$, etc. are the dynamic corrections to the dispersion equation. Accounting for these terms does not change the algebraic structure of the equation; it remains quadratic in $\mathbf{q}$ and its solutions remain similar to those in local, isotropic, homogeneous media. Generally, these solutions can be written as $q^{2}=\epsilon_{\text {eff }}(k) k^{2}$. However, if we account for the dynamic corrections, $\epsilon_{\mathrm{eff}}(k)$ will no longer satisfy the scaling laws discussed in section 7 of [1]. In particular, rescaling the composite as $\mathbf{r} \rightarrow \beta \mathbf{r}$ will change $\epsilon_{\text {eff }}(k)$; the law of unaltered ratios will also not hold.

Keeping the terms proportional to $\left(q_{p} h\right)^{2},\left(q_{p} h\right)^{4}$ or $q_{x}^{2} q_{y}^{2} q_{z}^{2} h^{6}$ (anisotropic terms of this form do not contradict the cubic symmetry), etc., will fundamentally change the algebraic form of the dispersion equation: it will become much more complicated than the quadratic equation characteristic of local and isotropic media. The dispersion equation can be written in this case as $q^{2}=\epsilon_{\text {eff }}(k, \mathbf{q}) k^{2}$. The explicit and sometimes very complicated dependence of $\epsilon_{\text {eff }}$ on $\mathbf{q}$ can be considered as an effect of spatial nonlocality of the constitutive relations.

Many important physical effects of nonlocality can be glimpsed from the dispersion equation. This includes additional waves, birefringence, and optical activity (in systems without a center of symmetry) [6]. Unfortunately, the dispersion equation does not give us the complete information about the medium. Indeed, solving boundary value problems in finite samples requires the knowledge of the influence function $f_{e}\left(\mathbf{r}, \mathbf{r}^{\prime} ; \tau\right)$ [the nonlocal generalization of the function $f_{e}(\mathbf{r}, \tau)$ that appears in (7a)]. But $f_{e}\left(\mathbf{r}, \mathbf{r}^{\prime} ; \tau\right)$ is not the spatial Fourier transform of $\epsilon_{\text {eff }}(k, \mathbf{q})$, nor is it a function of the shift $\mathbf{r}-\mathbf{r}^{\prime}$; the latter simplification is obtained only sufficiently far from the medium boundaries. Generally, spatial nonlocality and boundary value problems do not come together easily.

Things are further complicated by a wide-spread belief that weak nonlocality of the dielectric permittivity is physically indistinguishable from nontrivial magnetic response. For the precise statement of this equivalence principle and relevant references see [28]. In fact, the equivalence holds with respect to the dispersion relation but not the medium impedance. A detailed analysis of the equivalence principle is given in Ref. [29]. Here we only mention an obvious counterexample: an analytical solution can be easily obtained for a plane wave incident on a slab or a sphere with purely local $\epsilon$ and $\mu$. However, the same problem can not be solved so easily if the object has a nonlocal permittivity and $\mu=1$. If we define some function $f_{e}\left(\mathbf{r}, \mathbf{r}^{\prime} ; \tau\right)$ and solve somehow the resultant integro-differential equations, the electromagnetic fields scattered by the object will be not the 
same as in the first case.

The above discussion underscores the fundamental importance of what happens at the medium boundary. In section 3.A, we have already seen that disregard of the boundaries can yield an incorrect result even in very simple physical situations. In the more complicated scenarios considered by the extended homogenization theories, boundaries are crucial.

To illustrate the importance of boundaries one more time, consider an elementary but frequently overlooked example. If we know only the dispersion relation of an isotropic and local medium, there is no good way to define the parameters $\epsilon$ and $\mu$ separately. Indeed, the law of dispersion in this medium is $q^{2}=n^{2} k^{2}$ where $n^{2}=\epsilon \mu$. Assume that we have computed $n^{2}$. All we can gain from this knowledge is the product of $\epsilon$ and $\mu$, not these two quantities separately. This fact is frequently overlooked when extended homogenization theories are developed to predict both $\epsilon$ and $\mu$ but infinite unbounded media are considered. The main difficulty of the extended homogenization theories is accounting for the lack of translational invariance that occurs near the medium boundaries.

Now we have to backtrack a little and recall that, in the previous subsection, we have assumed without a justification that $\mu_{\text {eff }}=1$. This was, however, not a random guess. In the long wavelength limit, one can consider the medium with a planar boundary and compute the reflection and transmission coefficients [30]. Alternatively, one can compute the total magnetic moment of the medium in some simple geometry and apply the results of section 3.B. Both approaches will indicate that the correct choice is $\mu_{\text {eff }}=1$. Also, all experimental evidence tells us that there is no artificial magnetism in composites whose heterogeneity size is much smaller than the wavelength.

But beyond the long wavelength approximation, one enters a gray area in which the scaling laws discussed in section 7 of [1] do not work, the magnetic effects can be considered as important and introduction of a nontrivial effective magnetic permeability as feasible even in intrinsically non-magnetic composites. The big question is whether the medium can be reasonably characterized by local tensors $\hat{\epsilon}$ and $\hat{\mu}$ or, perhaps, by a more general but still local constitutive tensor. The extended (nonasymptotic) electromagnetic homogenization theories is a subject of active ongoing research [31-38]. The above references and many references therein will give an interested reader useful leads for further exploration of the subject.

\section{WAVES IN PHOTONIC CRYSTALS IN THE LOW FRE- QUENCY LIMIT}

We have seen in the previous section that the model of an infinite lattice of point polarizable particles is not well-behaved mathematically unless we assume implicitly or explicitly something about the particle shape and size. All methods of regularizing the divergent lattice sums boil down to making these assumptions, often in a disguised form. It makes sense therefore to abandon the point-particle model and consider the actual physical inclusions arranged periodically. We will see in this section that this approach is ultimately rewarding since it is free from any mathematical difficulties and allows one to account self-consistently for the effects of inclusions interaction, anisotropy, etc. After all, Maxwell's equations are not selfcontradictory and all mathematical difficulties grow from making ill-justified or unphysical approximations.

The mathematical developments shown in this section are not fundamentally different from those used for the case of

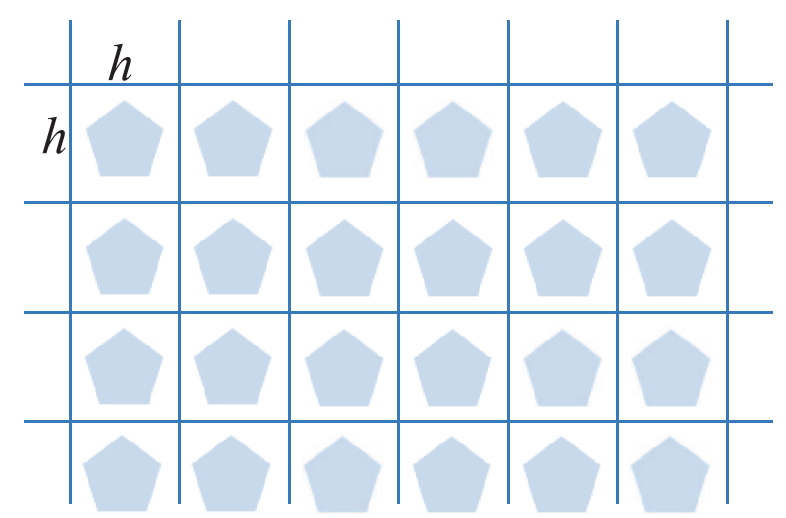

Fig. 3. (color online) A schematic illustration of a twocomponent photonic crystal. Identical inclusions are periodically arranged in a host medium. For simplicity, we assume that the host medium is vacuum. The pattern is threedimensional.

point polarizable particles. However, some more advanced algebraic manipulations will be used.

\section{A. Bloch waves in a photonic crystal}

Consider a three-dimensional periodic medium shown schematically in Fig. 3. nclusions of arbitrary shape are arranged periodically on an infinite cubic lattice of pitch $h$. We denote the elementary cells of the lattice by $\mathbb{C}_{n}$ and the center of $\mathbb{C}_{n}$ by $\mathbf{r}_{n}=h\left(n_{x}, n_{y}, n_{z}\right)$. As above, $n=\left(n_{x}, n_{y}, n_{z}\right)$ is a composite index.

Each elementary cell contains an inclusion. Let the spatial region occupied by the $n$-th inclusion be $\mathbb{V}_{n} \in \mathbb{C}_{n}$. All regions $\mathbb{V}_{n}$ are of exactly the same shape and the same volume $V$ and differ only by translations. In the figure, we have shown $\mathbb{V}_{n}$ as connected but this assumption is not important for us. Also, $\mathbb{V}_{n}$ can touch the boundary of $\mathbb{C}_{n}$ and the center of the cell, $\mathbf{r}_{n}$, can lie inside or outside of $\mathbb{V}_{n}$.

Further, we assume that the material of inclusions has the permittivity $\epsilon \neq 1$ and the material of the host is vacuum. Later on, we will generalize the consideration to the case of a twocomponent mixture by the usual substitution $\epsilon \rightarrow \epsilon_{i} / \epsilon_{h}$ and $\epsilon_{\text {eff }} \rightarrow \epsilon_{\text {eff }} / \epsilon_{h}$.

We start from the integral equation (30). Since we are looking for an eigen-solution in an infinite medium, we set the external field $\mathbf{E}_{\text {ext }}$ to zero. We also use the regularizing decomposition (40) for $\hat{G}\left(\mathbf{r}, \mathbf{r}^{\prime}\right)=\hat{F}\left(\mathbf{r}-\mathbf{r}^{\prime}\right)$ and obtain the equation

$$
\mathbf{E}(\mathbf{r})=-\frac{4 \pi}{3} \mathbf{P}(\mathbf{r})+\int \hat{F}_{R}\left(\mathbf{r}-\mathbf{r}^{\prime}\right) \mathbf{P}\left(\mathbf{r}^{\prime}\right) d^{3} r^{\prime} .
$$

This equation is valid everywhere in space. However, the function $\mathbf{P}(\mathbf{r})$ is nonzero only inside the inclusions. Since all inclusions are equivalent, it is possible to re-formulate the integral equation (63) so that it involves only one inclusion. Indeed, let us restrict attention to $\mathbf{r} \in \mathbb{V}_{n}$. We then multiply (63) by $(\epsilon-1) / 4 \pi$ and obtain after some additional re-arrangement

$$
\mathbf{P}(\mathbf{r})=\frac{3 \chi}{4 \pi} \sum_{m} \int_{\mathbf{r}^{\prime} \in \mathbb{V}_{m}} \hat{F}_{R}\left(\mathbf{r}-\mathbf{r}^{\prime}\right) \mathbf{P}\left(\mathbf{r}^{\prime}\right) d^{3} r^{\prime}, \quad \mathbf{r} \in \mathbb{V}_{n},
$$

where

$$
\chi=\frac{\epsilon-1}{\epsilon+2}
$$


is the spectral parameter of the theory. We will seek a solution to (64) in the form of a Bloch wave $\mathbf{P}(\mathbf{r})=\exp (i \mathbf{q} \cdot \mathbf{r}) \tilde{\mathbf{P}}(\mathbf{r})$, where $\mathbf{q}$ is the Bloch wave number (to be determined) and $\tilde{\mathbf{P}}(\mathbf{r})$ is a lattice-periodic function, which satisfies $\tilde{\mathbf{P}}\left(\mathbf{r}+\mathbf{r}_{n}\right)=\tilde{\mathbf{P}}(\mathbf{r})$ for any lattice vector $\mathbf{r}_{n}$.

If $\mathbf{r} \in \mathbb{V}_{n}$, we can make a change of variables $\mathbf{r}=\mathbf{r}_{n}+\mathbf{R}$ where $\mathbf{R} \in \mathbb{V}_{0} \in \mathbb{C}_{0}$ and $\mathbb{C}_{0}$ is the elementary cell centered around the point $\mathbf{r}_{0}=0$. Then the Bloch wave ansatz takes the form $\mathbf{P}(\mathbf{r})=\exp \left[i \mathbf{q} \cdot\left(\mathbf{r}_{n}+\mathbf{R}\right)\right] \tilde{\mathbf{P}}(\mathbf{R})$. Upon substitution of this expression into (64), we obtain

$$
\tilde{\mathbf{P}}(\mathbf{R})=\frac{3 \chi}{4 \pi} \int_{\mathbf{R}^{\prime} \in \mathbb{V}_{0}} \hat{W}\left(\mathbf{R}, \mathbf{R}^{\prime}\right) \tilde{\mathbf{P}}\left(\mathbf{R}^{\prime}\right) d^{3} R^{\prime}, \quad \mathbf{R} \in \mathbb{V}_{0},
$$

where

$$
\hat{W}\left(\mathbf{R}, \mathbf{R}^{\prime}\right)=\sum_{m} \hat{F}_{R}\left(\mathbf{r}_{n}-\mathbf{r}_{m}+\mathbf{R}-\mathbf{R}^{\prime}\right) e^{-i \mathbf{q} \cdot\left(\mathbf{r}_{n}-\mathbf{r}_{m}+\mathbf{R}-\mathbf{R}^{\prime}\right)} .
$$

Note that the right-hand side of (67) is independent of $n$ since all cells of an infinite lattice are equivalent.

Next, we substitute the Fourier representation (35) of $\hat{F}_{R}$ into the right-hand side of (67) and use the Poisson summation formula (52). This yields

$$
\hat{W}\left(\mathbf{R}, \mathbf{R}^{\prime}\right)=\frac{1}{h^{3}} \sum_{n} \hat{K}_{R}\left(\mathbf{q}+\mathbf{g}_{n}\right) e^{i \mathbf{g}_{n} \cdot\left(\mathbf{R}-\mathbf{R}^{\prime}\right)} .
$$

This expression still contains a summation over the reciprocal lattice vectors $\mathbf{g}_{n}=(2 \pi / h)\left(n_{x}, n_{y}, n_{z}\right)$. The advantage of using the expression (68) is the availability of a simple analytical expression for $\hat{K}_{R}(46)$.

We now wish to reformulate the integral equation (66) as an algebraic equation, which is amenable to numerical solution. To this end, we recall that $\tilde{\mathbf{P}}(\mathbf{R})$ is a lattice-periodic function and therefore it can be expanded as

$$
\tilde{\mathbf{P}}(\mathbf{R})=\sum_{n} \mathbf{P}_{n} e^{i \mathbf{g}_{n} \cdot \mathbf{R}} .
$$

Here $\mathbf{P}_{n}$ are the amplitudes of the Bloch harmonics; for a plane wave in a truly homogeneous medium, we have $\mathbf{P}_{n} \propto \delta_{n 0}$. By substituting the ansatz (69) into (66), we obtain an algebraic equation for the Bloch amplitudes:

$$
\mathbf{P}_{n}=f \frac{3 \chi}{4 \pi} \hat{K}_{R}\left(\mathbf{q}+\mathbf{g}_{n}\right) \sum_{m} M\left(\mathbf{g}_{n}-\mathbf{g}_{m}\right) \mathbf{P}_{m},
$$

where $f=V / h^{3}$ is the volume fraction of inclusions and $M(\mathbf{g})$ is the shape function; it is defined by the Fourier integral

$$
M(\mathbf{g})=\frac{1}{V} \int_{\mathbf{R} \in \mathbb{V}_{0}} e^{-i \mathbf{g} \cdot \mathbf{R}} d^{3} R .
$$

This integral can be computed analytically for many regular shapes.

Thus, we have written a set of algebraic equations (70) for the Bloch amplitudes $\mathbf{P}_{n}$. This equation should be viewed as an eigenproblem but not of a traditional kind. Assume that we have fixed the frequency $\omega$ and the shape of the inclusions. Then the parameter $\chi$ is also fixed ( $\chi$ is the only variable in the equation that depends on $\epsilon$ ). The volume fraction $f$ is also fixed and the function $M(\mathbf{g})$ is uniquely defined by the shape of the inclusions. Note that $M(\mathbf{g})$ is invariant under coordinate rescaling $\mathbf{r} \rightarrow \beta \mathbf{r}$. Then the set of all values of $\mathbf{q}$ for which (70) has a nontrivial solution forms the isofrequency surface. The word "surface" should not be understood literally because q can be complex. Generally, the vectors $\mathbf{q}$ that allow for nontrivial solutions of (70) fill a very complicated six-dimensional manifold $\Omega$. For each $\mathbf{q} \in \Omega$, there can be several distinct solutions to (70) we can refer to these solutions as to the polarization states.

\section{B. Long wavelength approximation}

Equation (70) is too complicated for analysis and we will now simplify it by using the long wavelength approximation. As was done in section 4.D, we will consider the equations for $\mathbf{P}_{0}$ (with $\mathbf{g}_{0}=0$ in the left-hand side) and $\mathbf{P}_{n}$ (with $\mathbf{g}_{n} \neq 0$ ) separately. We will use the limit (56) in the latter case and write

$$
\begin{aligned}
& \mathbf{P}_{0}=f \frac{3 \chi}{4 \pi} \hat{K}_{R}(\mathbf{q})\left[\mathbf{P}_{0}+\sum_{n \neq 0} M\left(-\mathbf{g}_{n}\right) \mathbf{P}_{n}\right], \\
& \mathbf{P}_{n}=f \chi \hat{Q}_{n}\left[M\left(\mathbf{g}_{n}\right) \mathbf{P}_{0}+\sum_{m \neq 0} M\left(\mathbf{g}_{n}-\mathbf{g}_{m}\right) \mathbf{P}_{m}\right], n \neq 0 .
\end{aligned}
$$

Here the index $n=0$ is equivalent to the triplet $\left(n_{x}, n_{y}, n_{z}\right)=$ $(0,0,0)$ and similarly for $m$.

In deriving (72), we have assumed that $k h,\left|q_{p}\right| h \ll 1$ or, equivalently, we have taken the limit $h \rightarrow 0$ under the condition that the proportions of all inclusions relative to the cell size are fixed. Eq. (72a) does not involve any approximations. The only place where the reciprocal lattice vectors (and the variable $h)$ appear in (72a) is $M\left(\mathbf{g}_{n}\right)$. But the functions $M(\mathbf{g})$ are invariant with respect to rescaling and, therefore, do not change when we take the limit $h \rightarrow 0$. In deriving (72b), we have encountered the expression $\hat{K}_{R}\left(\mathbf{q}+\mathbf{g}_{n}\right)$ with $\mathbf{g}_{n} \neq 0$, which depend on $h$. The limit of this term is given by (56).

The crucial observation that we need is this: $(72 b)$ is a set of linear equations with respect to the amplitudes $\mathbf{P}_{n}$ with $n \neq 0$ in which $\mathbf{P}_{0}$ can be viewed as a free term. To be sure, the set is infinite and any numerical solution will require truncation. But the important point is that we can view (72b) as an equation with a nonzero free term, not an eigenproblem. Then, from linearity, it follows that $\mathbf{P}_{n}=\hat{T}_{n} M\left(\mathbf{g}_{n}\right) \mathbf{P}_{0}$. The set of tensors $\hat{T}_{n}$ can be determined by solving the system of equations $(72 b)$ for three different right-hand sides: $\mathbf{P}_{0}=\mathbf{e}_{x}, \mathbf{P}_{0}=\mathbf{e}_{y}$ and $\mathbf{P}_{0}=\mathbf{e}_{z}$, where $\mathbf{e}_{p}(p=x, y, z)$ are unit vectors along the axes of a Cartesian frame. Consequently, we can write

$$
\sum_{n \neq 0} M\left(-\mathbf{g}_{n}\right) \mathbf{P}_{n}=\hat{\Sigma} \mathbf{P}_{0}
$$

where

$$
\hat{\Sigma}=\sum_{n \neq 0} M\left(-\mathbf{g}_{n}\right) \hat{T}_{n} M\left(\mathbf{g}_{n}\right)
$$

By substituting (73) into (72a), we find that the dispersion equation takes the form

$$
\left[\hat{I}-f \chi \frac{\left(2 k^{2}+q^{2}\right) \hat{I}-3 \mathbf{q} \otimes \mathbf{q}}{q^{2}-k^{2}}(\hat{I}+\hat{\Sigma})\right] \mathbf{P}_{0}=0,
$$

where we have used the explicit expression (46) for $\hat{K}_{R}(\mathbf{q})$.

The derivations of this section involved a couple of mathematically nontrivial steps, in particular, the splitting of the set of equations (70) into two subsets (72) and subsequent algebraic manipulations. It can be mentioned that the final result follows directly from the fundamental theorem of determinants of block matrices. Here we have followed a less formal approach.

We conclude the subsection with a few remarks.

(i) The tensor $\hat{\Sigma}$ depends on the shape and orientation of inclusions and on the spectral parameter $\chi$ and therefore on $\epsilon$.

(ii) The tensor $\hat{\Sigma}$ can be computed by solving a well-defined system of algebraic equations on a computer. Theoretically, the 
set is infinite but we can truncate it by restricting the reciprocal lattice vectors to the cubic box with excluded center $\mathbb{B}(L)$, which was defined in section 4.D. Convergence can be investigated by repeatedly increasing the box size $L$, e.g., by the factor of 2 .

(iii) In general, the tensor $\hat{\Sigma}$ is complex symmetric and not necessarily diagonalizable by an orthogonal transformation. If it is not, then there is no Cartesian frame in which $\hat{\Sigma}$ is diagonal. We have not considered this (perhaps, unusual) case and will not discuss it any further.

(iv) Note the limit $\lim _{f \rightarrow 1} \hat{\Sigma}=0$. However, $\lim _{f \rightarrow 0} \hat{\Sigma}=\hat{\Sigma}_{0}$ where $\hat{\Sigma}_{0}$ is zero for spherical inclusions but not for other shapes.

\section{The mixing formula}

The dispersion equation (75) has nontrivial solutions if the determinant of the $3 \times 3$ matrix in the square brackets is zero. The particular values of $\mathbf{q}$ for which this is the case are the Bloch wave vectors. There can be several distinct polarization states corresponding to a given Bloch wave vector. The consideration is rather involved mathematically for a general anisotropic material. We will derive therefore the homogenization result for an isotropic composite and then write without proof a more general formula applicable to the case when $\hat{\Sigma}$ is tensorial but diagonal in some Cartesian frame.

The composite is isotropic for any inclusions that obey the same cubic symmetry as the lattice. For example, such inclusions can be cubes or spheres centered around the points $\mathbf{r}_{n}$. However, much more complicated shapes can also obey the cubic symmetry. The major simplification that one obtains in this case is $\hat{\Sigma}=\Sigma \hat{I}$, where $\Sigma$ is a scalar. Then there are two linearlyindependent transverse polarization states with $\mathbf{q} \cdot \mathbf{P}_{0}=0$ for each q. A longitudinal polarization state (such that $\mathbf{P}_{0} \| \mathbf{q}$ ) exists only if $\epsilon=1-3 /[2 f(1+\Sigma)+1]$. Usually, this equality does not hold and we can safely ignore longitudinal waves. Under these conditions, equation (75) is simplified to

$$
1-f \chi \frac{2 k^{2}+q^{2}}{q^{2}-k^{2}}(1+\Sigma)=0 .
$$

We now introduce the effective permittivity through the relation $q^{2}=\epsilon_{\text {eff }} k^{2}$, use the explicit definition of $\chi(65)$ and transform (76) to

$$
\frac{\epsilon_{\mathrm{eff}}-1}{\epsilon_{\mathrm{eff}}+2}=f \frac{\epsilon-1}{\epsilon+2}(1+\Sigma) .
$$

We finally generalize the above result for the case of a twocomponent mixture. To this end, we apply the usual transformation $\epsilon_{\mathrm{eff}} \rightarrow \epsilon_{\mathrm{eff}} / \epsilon_{h}, \epsilon \rightarrow \epsilon_{i} / \epsilon_{h}$ and obtain the familiarlylooking formula

$$
\frac{\epsilon_{\mathrm{eff}}-\epsilon_{h}}{\epsilon_{\mathrm{eff}}+2 \epsilon_{h}}=f \frac{\epsilon_{i}-\epsilon_{h}}{\epsilon_{i}+2 \epsilon_{h}}(1+\Sigma) .
$$

It should be kept in mind that the spectral parameter $\chi$ in equation (72b) (which is used to compute $\Sigma$ ) should also be changed to $\chi=\left(\epsilon_{i}-\epsilon_{h}\right) /\left(\epsilon_{i}+2 \epsilon_{h}\right)$.

If not for the factor $\Sigma$, equation (78) would be the Maxwell Garnett mixing formula written in the form (F25). A nonzero factor $\Sigma$ accounts for the deviation of the rigorous homogenization result from the Maxwell Garnett prediction.

Just like the Maxwell Garnett mixing formula, (78) is not symmetric and, in fact, we do not expect the rigorous homogenization result to be symmetric. The definition of the mixing formula symmetry and a more detailed discussion are given in section 3 of [1]. Equation (78) captures correctly the effects of shape of the inclusions and therefore it is not symmetric.

Another interesting feature of (78) is this. Consider cubic inclusions. Then we can change the size of the inclusion from 0 to $h$ and it will correspond to changing $f$ from 0 to 1 . At $f=0$ we have $\epsilon_{\text {eff }}=\epsilon_{h}$, and at $f=1$ we have $\epsilon_{\text {eff }}=\epsilon_{i}$. If we plot $\epsilon_{\text {eff }}(f)$ given by (78) parametrically in the complex $\epsilon$-plane, we will obtain a smooth curve that connects the points $\epsilon_{h}$ and $\epsilon_{i}$ and lies inside the Wiener bounds. In fact, there are two different curves that connect $\epsilon_{h}$ and $\epsilon_{i}$ in this manner, depending on whether we assume that $f=0$ corresponds to a homogeneous medium with the permittivity $\epsilon_{h}$ [as is assumed in Eq. (78)] or that it corresponds to a homogeneous medium with the permittivity $\epsilon_{i}$. The two curves will not intersect except at the ends, which is a manifestation of the lack of symmetry of the mixing formula. The Maxwell Garnett approximation has exactly the same property.

Therefore, (78) is a corrected or a generalized version of the isotropic Maxwell Garnett mixing formula, which accounts properly for the electromagnetic interaction of inclusions (in a periodic system) but has the same general mathematical properties as the classical mixing formula.

We now state without proof the result for the case when $\hat{\Sigma}$ is diagonal in some Cartesian frame $X Y Z$ but its principal values are different. The result is rigorously derived in Ref. [30] and is a straightforward generalization of (78), viz,

$$
\frac{\left(\epsilon_{\text {eff }}\right)_{p}-\epsilon_{h}}{\left(\epsilon_{\text {eff }}\right)_{p}+2 \epsilon_{h}}=f \frac{\epsilon_{i}-\epsilon_{h}}{\epsilon_{i}+2 \epsilon_{h}}\left(1+\Sigma_{p}\right), \quad p=x, y, z .
$$

Here $\Sigma_{p}$ is one of the principal values of the tensor $\hat{\Sigma}$. Solving for $\left(\epsilon_{\text {eff }}\right)_{p}$, we obtain the mixing formula:

$$
\begin{aligned}
\left(\epsilon_{\mathrm{eff}}\right)_{p}=\epsilon_{h} \frac{1+2 f \frac{\epsilon_{i}-\epsilon_{h}}{\epsilon_{i}+2 \epsilon_{h}}\left(1+\Sigma_{p}\right)}{1-f \frac{\epsilon_{i}-\epsilon_{h}}{\epsilon_{i}+2 \epsilon_{h}}\left(1+\Sigma_{p}\right)} \\
=\epsilon_{h} \frac{\epsilon_{h}+\frac{1+2 f\left(1+\Sigma_{p}\right)}{3}\left(\epsilon_{i}-\epsilon_{h}\right)}{\epsilon_{h}+\frac{1-f\left(1+\Sigma_{p}\right)}{3}\left(\epsilon_{i}-\epsilon_{h}\right)}
\end{aligned}
$$

This is the rigorous homogenization result for anisotropic composites that have three well-defined optical axes.

The second expression in (80) can be compared to the classical anisotropic Maxwell Garnett mixing formula (FP34). It can be seen that the two formulas can not be made equivalent by some special choice of the depolarization factors $v_{p}$. Indeed, the condition of equivalence of the two expressions is

$$
v_{p}=\frac{1}{3}\left(1-\frac{\Sigma_{p}}{1+\Sigma_{p}} \frac{1}{\chi}\right), \text { where } \chi=\frac{\epsilon_{i}-\epsilon_{h}}{\epsilon_{i}+2 \epsilon_{h}} .
$$

But in the Maxwell Garnett theory, $v_{p}$ is a purely geometrical parameter, which is independent of $\epsilon_{i}$ and $\epsilon_{h}$. Eq. (81) can hold only if $\Sigma_{p}=\beta_{p} \chi /\left(1-\beta_{p} \chi\right)$, where $\beta_{p}$ is a materialindependent constant. If the latter equality holds, then it follows from (81) that $v_{p}=\left(1-\beta_{p}\right) / 3$.

In other words, for the Maxwell Garnett approximation to be precise, it is required that $\hat{\Sigma}$ has only one resonance for a given polarization with the resonance value of $\chi$ being $1 / \beta_{p}=$ $1 /\left(1-3 v_{p}\right)$. But this property does not generally hold except if $\left(\epsilon_{\text {eff }}\right)_{p}$ lies on one of the Wiener bounds, that is, for composites of some very special geometry. Of course, on the Wiener 
bounds, the anisotropic Maxwell Garnett mixing formula is precise. But in a more general case, the functional dependence of the form $\Sigma_{p}(\chi)=\sum_{n} \beta_{n p} \chi /\left(1-\beta_{n p} \chi\right)$ is valid, and the sum is not reduced to just one term. This follows from the algebraic form of Eq. (72b), which determines $\hat{\Sigma}(\chi)$. Therefore, in reality, the function $\hat{\Sigma}(\chi)$ has multiple resonances. The physical significance of the resonance values of $\chi, \chi_{n p}=1 / \beta_{n p}$ is the following. For $\chi$ in the vicinity of one of its resonance values, electromagnetic interaction of inclusions becomes very strong. In a sense, the Maxwell Garnett approximation assumes that only one such resonance exists. If this is not so, the approximation can break down dramatically.

We can conclude that the Maxwell Garnett's treatment of anisotropy is based on a single geometrical parameter $v_{p}$ while, in fact, the rigorous description involves many such factors ( $\beta_{n p}$, using the above notations) or the function $\Sigma_{p}$ that depends on both geometry and composition. The ambiguity of the anisotropic formula discussed in section 4 of [1] is mathematically related to this fact. In the next section, we will show an example of anisotropy that is not captured accurately by the Maxwell Garnett theory.

\section{NUMERICAL EXAMPLES}

Let us truncate the infinite set of equations $(72 b)$ by restricting the reciprocal lattice vectors to the box with excluded center $\mathbb{B}(L)$. In this case, the number of equations that must be solved to compute $\hat{\Sigma}$ is $N=3\left[(2 L+1)^{3}-1\right]$. Note that the equations must be solved with three different right-hand sides to gain access to all elements of $\hat{\Sigma}$.

It can be seen that the number of equations scales as $O\left(L^{3}\right)$. This makes direct solution of $3 \mathrm{D}$ problems difficult and we must resort to iterative methods. One such fast-converging iterative algorithm is based on a continued-fraction expansion [30]. The method is spectral, which means that the computationallyintensive part of this algorithm is independent of the spectral parameter $\chi$ and, therefore, of $\epsilon_{i}$ and $\epsilon_{h}$. This can be useful if we are interested in the spectral dependence of $\epsilon_{\text {eff }}(\omega)$ for a fixed geometry of the composite and dispersive $\epsilon_{h}(\omega)$ and $\epsilon_{i}(\omega)$. However, in the examples shown below, we fix $\epsilon_{h}$ and $\epsilon_{i}$ and compute $\epsilon_{\text {eff }}(f)$ by changing the size of the inclusions relative to $h$ up to the geometrical limit. In this case, spectral methods are not computationally advantageous. Nevertheless, the iterative method mentioned above is efficient enough to handle 3D problems. Below, numerical results will be shown for $L=32$. Convergence was verified by doubling the box size $L$ in some of the cases and repeating the computations with $L=64$.

Simulations were performed for spherical and cubic inclusions and for two types of parallelepipeds shown in Fig. 4. Numerical results are shown in Fig. 5. We have used the same permittivities of the composite constituents as in the first part of this tutorial (in section 6): $\epsilon_{1}=1.5+1.0 i$ and $\epsilon_{2}=-4.0+2.5 i$. We now briefly describe the results.

In Panel (a) we show the effective permittivity of a composite consisting of spherical inclusions of the radius $a$ that varies from zero to the point of touching, $a=h / 2$. The composite is isotropic. The data points computed according to the formula (80) are shown by circles. We consider two cases: metal inclusions in the dielectric host and vice versa. Rigorous homogenization results are compared to the Maxwell Garnett and Bruggeman's mixing formulas. Of course, in the analytical mixing formulas $f$ is not limited by the geometry and we have shown the respective curves for $0 \leq f \leq 1$. As was mentioned in [1], these
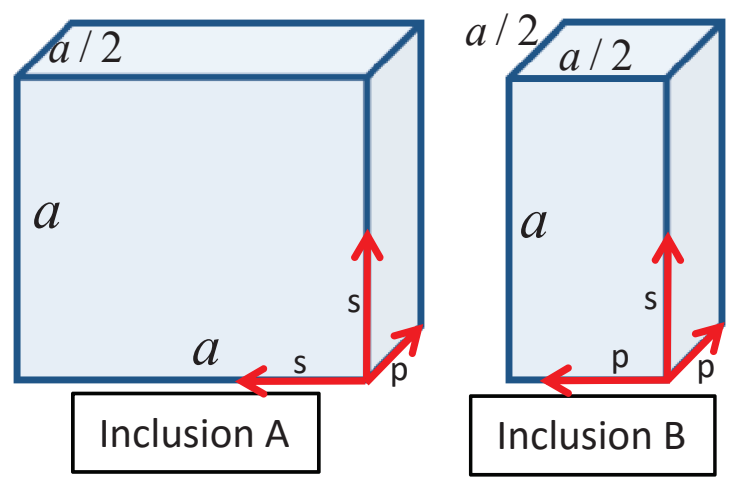

Fig. 4. Illustration of the anisotropic inclusions and polarization labels used in the simulations shown in Fig. 5(c) below.

curves always connect the two points $\epsilon_{1}$ and $\epsilon_{2}$ in the complex$\epsilon$ plane. However, the value of $f$ for the rigorously-computed numerical data are limited by the condition of non-intersection of the inclusions. Due to this reason, the numerical data terminate inside the Wiener bounds. The last data point corresponds to touching spheres.

It can be seen that the Maxwell Garnett formula is accurate at low-to-moderate volume fractions while the Bruggeman's formula does not provide a meaningful correction. Note that the comparison of the numerical results and analytical approximations in Fig. 5 is not quite definitive: even if two points overlap in the complex- $\epsilon$ plane, they can correspond to different values of $f$. Still, it is true that the Maxwell Garnett mixing formula provides a good approximation for spherical inclusions. Admittedly, we did not consider the case of strong electromagnetic resonances. The materials considered have large imaginary parts, which suppress the resonant phenomena.

In Panel (b) we show similar data for cubes. Now the volume fraction $f$ can vary from 0 to 1 in the analytical expressions as well as for the actual inclusions. Again, the Maxwell Garnett mixing formula yields surprisingly accurate results while the Bruggeman's formula can be seen as providing a meaningful correction at low volume fractions and then breaking down completely.

The most interesting cases are shown in Panel (c). Here the inclusions are parallelepipedal as shown in Fig. 4. The effective medium is anisotropic and uniaxial. We show all principal values of the effective permittivity tensor. In the case of inclusion A, the geometrical limit of touching inclusions corresponds to a one-dimensional layered medium of alternating metal and dielectric layers. We know that the effective permittivity of this medium lies on one of the Wiener bounds. This is indeed the case. Just as expected, the effective permittivity starts from $\epsilon_{1}$ or $\epsilon_{2}$ (depending on the choice of the host) at $f=0$ and then terminates at the linear or circular Wiener bound for the s- or $\mathrm{p}$ polarization, respectively, at $f=0.5$. Moreover, at $f=0.5$, the layered medium is invariant under the permutation $\epsilon_{1} \leftrightarrow \epsilon_{2}$. Therefore, the curves AsDH and AsMH start from $\epsilon_{1}$ and $\epsilon_{2}$ at $f=0$ and then meet at the linear Wiener bound at $f=0.5$. Analogously, the curves ApDH and ApMH meet at the circular Wiener bound at $f=0.5$. This behavior can not be captured by the Maxwell Garnett or Bruggeman's mixing formulas, which always produce curves that connect $\epsilon_{1}$ and $\epsilon_{2}$ without touching the Wiener bounds. The analytical predictions are not plotted 


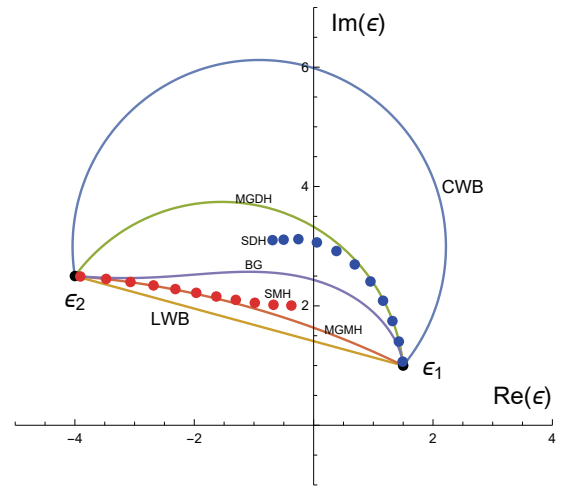

(a)

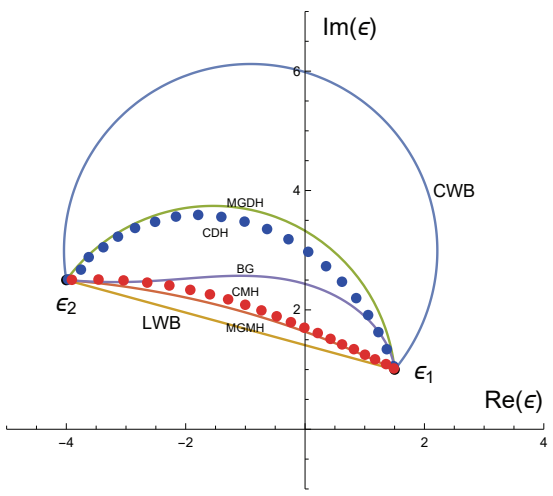

(b)

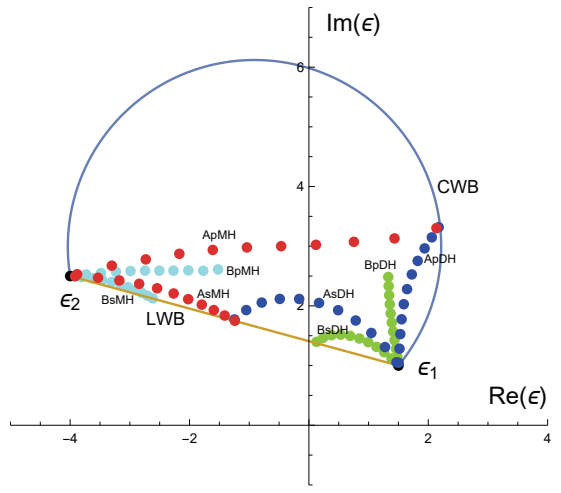

(c)

Fig. 5. Numerical and analytical results for the effective permittivity of different composites. The inclusions are spherical (a), cubic (b) and parallelepipedal (c). The shapes of the parallelepipeds and the corresponding polarization labels (directions of the fundamental Bloch harmonic $\mathbf{P}_{0}$ ) as shown in Fig. 4 . The volume fraction of the inclusions is varied from zero to the maximum value $f_{\text {max }}$ allowed by the geometry. In particular, $f_{\max }=\pi / 6(\mathrm{a}), f_{\max }=1(\mathrm{~b})$ and $f_{\max }=0.5$ (c), inclusion $\mathrm{A}$ and $f_{\max }=0.25$ (c), inclusion B. Results are shown for both cases when $\epsilon_{h}=\epsilon_{1}, \epsilon_{i}=\epsilon_{2}$ (dielectric host) and $\epsilon_{h}=\epsilon_{2}, \epsilon_{i}=\epsilon_{1}$ (metal host). The labeling of various curves and data sets is of the form $X$ or XY or XvY. Here $X$ takes the values $X=M G$ (isotropic Maxwell Garnett mixing formula), $X=B G$ (isotropic Bruggeman's mixing formula), $X=S$ (Eq. (80) for spherical inclusions), $X=C$ (Eq. (80) for cubic inclusions) and $\mathrm{X}=\mathrm{A}, \mathrm{B}$ (Eq. (80) for parallelepipedal inclusions $\mathrm{A}$ or B shown in Fig. 4). The polarization label takes two values $\mathrm{v}=\mathrm{s}$ or $\mathrm{v}=\mathrm{p}$ and is applicable only to $\mathrm{X}=\mathrm{A}, \mathrm{B}$ (see Fig. 4). Finally, the label $\mathrm{Y}$ takes two values: $\mathrm{Y}=\mathrm{DH}$ for dielectric host and $\mathrm{Y=MH}$ for metal host. LWB and CWB are the linear and circular Wiener bounds. Note that some data points shown as circles were computed for the values of $f$ slightly different from the exact limiting values (for example, $f=0.01$ instead of $f=0$ ) to avoid complete overlap and obscuration of two or more data points.

in Panel (c) because we know a priori that these predictions can not be possibly accurate.

As for the inclusion B, its geometrical limit is a set of infinite rods of a square cross section and the volume fraction $f_{\max }=0.25$. For the s-polarization, the electric field in this medium is smooth (see [1], section 5). We therefore expect the corresponding values of the effective permittivity to lie on the linear Wiener bound. However, the composite is not invariant under the permutation $\epsilon_{1} \leftrightarrow \epsilon_{2}$. Therefore, the curves BsDH and $\mathrm{BsMH}$ indeed terminate at the linear Wiener bound but at different points. For the p-polarization, the displacement field D is not smooth. Therefore, the curves BpDH and BpMH stay well inside the Wiener bounds and do not touch the circular bound at $f=f_{\max }$.

\section{CONCLUDING REMARKS}

As was promised in the first part of this tutorial, we have derived a rigorous homogenization formula by considering a general photonic crystal in the long wavelength limit. The result is not entirely analytical. The homogenization formula contains a tensor $\hat{\Sigma}$, which is a function of the contrast and geometry and must be computed numerically. Setting $\hat{\Sigma}=0$ results in the isotropic Maxwell Garnett mixing formula. The anisotropic formula is obtained if the principal values of $\hat{\Sigma}$ are of the special form $\Sigma_{p}(\chi)=\beta_{p} \chi /\left(1-\beta_{p} \chi\right)$ where $\beta_{p}$ are constants. Here $\chi=\left(\epsilon_{i}-\epsilon_{h}\right) /\left(\epsilon_{i}+2 \epsilon_{h}\right)$ is the spectral parameters of the theory. We can identify the depolarization factors appearing in the classical formula as $v_{p}=\left(1-\beta_{p}\right) / 3$. If $\beta_{p}=0, v_{p}=1 / 3$ and the classical isotropic mixing formula is obtained.

However, there is a class of composites that are isotropic but whose rigorous effective permittivity is not equal to the Maxwell Garnett's prediction. For these composites, a more general functional dependence of the form $\Sigma_{p}(\chi)=$ $\sum_{n} \beta_{n p} \chi /\left(1-\beta_{n p} \chi\right)$ is valid, where the sum is not reduced to just one term. The composite is isotropic if $\Sigma_{p}$ is independent of $p$. This is a more general condition than the assumption that $\beta_{n p}=0$, which is, essentially, the sufficient and necessary condition for the isotropic Maxwell Garnett mixing formula to be precise. In general, the Maxwell Garnett-type family of approximations assume that there is only one resonance of the function $\Sigma_{p}(\chi)$. In actual composites, several resonances can be present simultaneously for any given polarization.

The problem we have considered in section 5 is known in the homogenization literature as the cell problem. There exist many mathematical formulations of the cell problem and many different approaches to its solutions. The specific approach of section 5 utilizes the basis of plane waves. It also reduces the numerical part to computing the tensor $\hat{\Sigma}$, which was discussed above. Other approaches may use local bases or compute the effective permittivity directly, without casting it first in a special form resembling the Maxwell Garnett mixing formula.

When we considered waves on lattices of polarized particles or in photonic crystals, we have used integral equations as the mathematical point of departure in order to arrive at the desired result in the most direct and economical way. However, there is nothing special in this approach. One can as well start from the differential equations and arrive at exactly the same result. Still, the fact that the Maxwell Garnett mixing formula seems to be "embedded" in the integral equations of classical electrodynamics is quite remarkable and we have intentionally used the integral equations to emphasize this point.

The numerical examples of section 6 show that the isotropic Maxwell Garnett mixing formula is quite robust and can work reasonably well even beyond the limit of very low volume fractions. Of course, this statement only concerns composites without strong resonance phenomena (which we have not discussed in the tutorial) and should not be understood too generally.

Finally, many of the ideas discussed in this tutorial (espe- 
cially, in the first part), and many interesting and relevant discussions, in particular, of the concept of the local field, can be found in the book [39]. Unfortunately, as of this writing, this book has not been translated into English.

\section{ACKNOWLEDGMENT}

This work has been carried out thanks to the support of the A*MIDEX project (No. ANR-11-IDEX-0001-02) funded by the "Investissements d'Avenir" French Government program, managed by the French National Research Agency (ANR).

\section{REFERENCES}

1. V. A. Markel, "Introduction to the Maxwell Garnett approximation: tutorial," J. Opt. Soc. Am. A 33, 1244-1256 (2016).

2. A. Chipouline, C. Simovski, and S. Tretyakov, "Basics of averaging of the Maxwell equations for bulk materials," Metamaterials 6, 77-120 (2012).

3. A. P. Vinogradov and A. M. Merzlikin, "Comment on "Basics of averaging of the Maxwell equations for bulk materials"," Metamaterials 6 , 121-125 (2012).

4. R. Resta and D. Vanderbilt, Physics of Ferroelectrics: A Modern Perspective (Springer, 2007), chap. Theory of polarization: A modern approach, pp. 31-68.

5. A notable exception is the Aharonov-Bohm effect, which can not be understood within the classical electromagnetic theory.

6. V. Agranovich and V. Ginzburg, Spatial Dispersion in Crystal Optics and the Theory of Excitons (Wiley-Interscience, New York, 1966).

7. L. D. Landau and L. P. Lifshitz, Electrodynamics of Continuous Media (Pergamon Press, Oxford, 1984).

8. H. G. Tompkins and W. A. McGahan, eds., Spectroscopic Ellipsometry and Reflectometry: A User's Guide (Wiley, 1999).

9. R. D. King-Smith and D. Vanderbilt, "Theory of polarization of crystalline solids," Phys. Rev. B 47, 1651-1654 (1993).

10. R. Resta, "Macroscopic electric polarization as a geometric quantum phase," Europhys. Lett. 22, 133-138 (1993).

11. D. Vanderbilt and R. D. King-Smith, "Electric polarization as a bulk quantity and its relation to surface charge," Phys. Rev. B 48, 4442-4455 (1993).

12. R. Resta, "Macroscopic polarization in crystalline dielectrics: The geometrical phase approach," Rev. Mod. Phys. 66, 899 (1994).

13. N. A. Spaldin, "A beginner guide to the modern theory of polarization," J. Sol. St. Chem. 195, 2-10 (2012).

14. R. Resta, "Electrical polarization and orbital magnetization: the modern theories," J. Phys.: Condens. Matter 22, 123201 (2010).

15. R. Bianco and R. Resta, "Orbital magnetization as a local property," Phys. Rev. Lett. 110, 087202 (2013).

16. G. D. Mahan and G. Obermair, "Polaritons at surfaces," Phys. Rev. 183, 834-841 (1969).

17. J. E. Sipe and J. Van Kranendonk, "Macroscopic electromagnetic theory of resonant dielectrics," Phys. Rev. A 9, 1806-1822 (1974).

18. W. Lamb, D. M. Wood, and N. W. Ashcroft, "Long-wavelength electromagnetic propagation in heterogeneous media," Phys. Rev. B 21, 22482266 (1980).

19. B. T. Draine and J. Goodman, "Beyond Clausius-Mossotti: Wave propagation on a polarizable point lattice and the discrete dipole approximation," Astrophys. J. 405, 685-697 (1993).

20. P. A. Belov and C. R. Simovski, "Homogenization of electromagnetic crystals formed by uniaxial resonant scatterers," Phys. Rev. E 72, 026615 (2005).

21. F. J. G. Abajo, "Light scattering by particle and hole arrays," Rev. Mod. Phys. 79, 1267-1290 (2007).

22. B. T. Draine, "The discrete-dipole approximation and its application to interstellar graphite grains," Astrophys. J. 333, 848-872 (1988).

23. V. A. Markel, "Scattering of light from two interacting spherical particles," J. Mod. Opt. 39, 853-861 (1992).
24. M. A. Yurkin, Handbook of Molecular Plasmonics (Pan Stanford Pub., Singapore, 2013), chap. Computational approaches for plasmonics, pp. 83-135.

25. P. de Vries, D. V. van Coevorden, and A. Lagendijk, "Point scatterers for classical waves," Rev. Mod. Phys. 70, 447-466 (1998).

26. B. T. Draine, Light Scattering by Nonspherical Particles (Academic Press, 2000), chap. The discrete dipole approximation for light scattering by irregular targets, pp. 226-273.

27. J. M. Borwein, M. L. Glasser, R. C. McPhedran, J. G. Wan, and I. J. Zucker, Lattice Sums Then and Now (Cambridge, 2013).

28. V. M. Agranovich and Y. N. Gartstein, "Electrodynamics of metamaterials and the Landau-Lifshitz approach to the magnetic permeability," Metamaterials 3, 1-9 (2009).

29. V. A. Markel and I. Tsukerman, "Current-driven homogenization and effective medium parameters for finite samples," Phys. Rev. B 88, 125131 (2013).

30. V. A. Markel and J. C. Schotland, "Homogenization of Maxwell's equations in periodic composites," Phys. Rev. E 85, 066603 (2012).

31. U. C. Hasar, G. Buldu, M. Bute, J. J. Barroso, T. Karacali, and M. Ertugrul, "Determination of constitutive parameters of homogeneous metamaterial slabs by a novel calibration-independent method," AIP Advances 4, 107116 (2014).

32. T. D. Karamanos, S. D. Assimonis, A. I. Dimitriadis, and N. V. Kantartzis, "Effective parameter extraction of 3D metamaterial arrays via first-principle homogenization theory," Photonics and Nanostructures 12, 291 (2014).

33. N. C. J. Clausen, S. Arslanagic, and O. Breinbjerg, "Comparison of spatial harmonics in infinite and finite Bragg stacks for metamaterial homogenization," Photonics and Nanostructures 12, 419 (2014).

34. A. Ciattoni and C. Rizza, "Nonlocal homogenization theory in metamaterials: Effective electromagnetic spatial dispersion and artificial chirality," Phys. Rev. B 91, 184207 (2015).

35. V. Sozio, A. Vallecchi, M. Albani, and F. Capolino, "Generalized Lorentz-Lorenz homogenization formulas for binary lattice metamaterials," Phys. Rev. B 91, 205127 (2015).

36. A. A. Shcherbakov and A. V. Tishchenko, "3D periodic dielectric composite homogenization based on the generalized source method," J. Opt. 17, 065101 (2015).

37. A. A. Krokhin, J. Arriaga, L. N. Gumen, and V. P. Drachev, "Highfrequency homogenization for layered hyperbolic metamaterials," Phys. Rev. B 93, 075418 (2016).

38. M. Caleap and W. Drinkwater, "Metamaterials: supra-classical dynamic homogenization," New J. Phys. 17, 123022 (2016).

39. A. P. Vinogradov, Electrodynamics of Composite Materials (URSS, Moscow, 2001). 